\title{
Importance of correlations and fluctuations on the initial source eccentricity in high-energy nucleus-nucleus collisions
}

\author{
B. Alver, ${ }^{4}$ B. B. Back, ${ }^{1}$ M. D. Baker, ${ }^{2}$ M. Ballintijn, ${ }^{4}$ D. S. Barton, ${ }^{2}$ R. R. Betts, ${ }^{7}$ R. Bindel,${ }^{8}$ W. Busza,${ }^{4}$ V. Chetluru, ${ }^{7}$
} E. García, ${ }^{7}$ T. Gburek, ${ }^{3}$ J. Hamblen, ${ }^{9}$ U. Heinz, ${ }^{6}$ D. J. Hofman, ${ }^{7}$ R. S. Hollis, ${ }^{7}$ A. Iordanova, ${ }^{7}$ W. Li,${ }^{4}$ C. Loizides ${ }^{4}$ S. Manly,${ }^{9}$ A. C. Mignerey, ${ }^{8}$ R. Nouicer, ${ }^{2}$ A. Olszewski ${ }^{3}$ C. Reed ${ }^{4}$ C. Roland,${ }^{4}$ G. Roland, ${ }^{4}$ J. Sagerer, ${ }^{7}$ P. Steinberg, ${ }^{2}$ G. S. F. Stephans ${ }^{4}$ M. B. Tonjes, ${ }^{8}$ A. Trzupek,${ }^{3}$ G. J. van Nieuwenhuizen, ${ }^{4}$ S. S. Vaurynovich, ${ }^{4}$ R. Verdier, ${ }^{4}$ G. I. Veres, ${ }^{4}$ P. Walters, ${ }^{9}$ E. Wenger, ${ }^{4}$ B. Wosiek, ${ }^{3}$ K. Woźniak, ${ }^{3}$ and B. Wyslouch ${ }^{4}$

${ }^{1}$ Physics Division, Argonne National Laboratory, Argonne, Illinois 60439-4843, USA

${ }^{2}$ Physics and C-A Departments, Brookhaven National Laboratory, Upton, New York 11973-5000, USA

${ }^{3}$ Institute of Nuclear Physics, Kraków, Poland

${ }^{4}$ Laboratory for Nuclear Science, Massachusetts Institute of Technology, Cambridge, Massachusetts 02139-4307, USA

${ }^{5}$ Department of Physics, National Central University, Chung-Li, Taiwan

${ }^{6}$ Department of Physics, Ohio State University, Columbus, Ohio 43210, USA

${ }^{7}$ Department of Physics, University of Illinois at Chicago, Chicago, Illinois 60607-7059, USA

${ }^{8}$ Department of Chemistry, University of Maryland, College Park, Maryland 20742, USA

${ }^{9}$ Department of Physics and Astronomy, University of Rochester, Rochester, New York 14627, USA

(Received 23 November 2007; published 31 January 2008)

\begin{abstract}
In relativistic heavy-ion collisions, anisotropic collective flow is driven, event by event, by the initial eccentricity of the matter created in the nuclear overlap zone. Interpretation of the anisotropic flow data thus requires a detailed understanding of the effective initial source eccentricity of the event sample. In this paper, we investigate various ways of defining this effective eccentricity using the Monte Carlo Glauber (MCG) approach. In particular, we examine the participant eccentricity, which quantifies the eccentricity of the initial source shape by the major axes of the ellipse formed by the interaction points of the participating nucleons. We show that reasonable variation of the density parameters in the Glauber calculation, as well as variations in how matter production is modeled, do not significantly modify the already established behavior of the participant eccentricity as a function of collision centrality. Focusing on event-by-event fluctuations and correlations of the distributions of participating nucleons, we demonstrate that, depending on the achieved event-plane resolution, fluctuations in the elliptic flow magnitude $v_{2}$ lead to most measurements being sensitive to the root-mean-square rather than the mean of the $v_{2}$ distribution. Neglecting correlations among participants, we derive analytical expressions for the participant eccentricity cumulants as a function of the number of participating nucleons, $N_{\text {part }}$, keeping nonnegligible contributions up to $\mathcal{O}\left(1 / N_{\text {part }}^{3}\right)$. We find that the derived expressions yield the same results as obtained from mixed-event MCG calculations which remove the correlations stemming from the nuclear collision process. Most importantly, we conclude from the comparison with MCG calculations that the fourth-order participant eccentricity cumulant does not approach the spatial anisotropy obtained assuming a smooth nuclear matter distribution. In particular, for the $\mathrm{Cu}+\mathrm{Cu}$ system, these quantities deviate from each other by almost a factor of 2 over a wide range in centrality. This deviation reflects the essential role of participant spatial correlations in the interaction of two nuclei.
\end{abstract}

DOI: 10.1103/PhysRevC.77.014906

PACS number(s): 25.75.Dw, 25.75.Gz

\section{INTRODUCTION}

One of the strongest pieces of evidence for the formation of a thermalized dense state of unconventional strongly interacting matter in ultrarelativistic nucleus-nucleus collisions at the BNL Relativistic Heavy Ion Collider (RHIC) [1-4] stems from the strong anisotropic collective flow measured in noncentral collision events [5-12]. Studies of the final charged particle momentum distributions have revealed strong collective effects in the form of anisotropies in the azimuthal distribution transverse to the direction of the colliding nuclei, and theory holds that their anisotropy around the beam axis in noncentral collisions is established during the earliest stages of the evolution of the collision fireball [13-16]. The main component of this anisotropy is called "elliptic flow" and its strength is commonly quantified by the second coefficient, $v_{2}$, in the Fourier decomposition of the azimuthal momentum distribution of observed particles relative to the reaction plane [17].

By now there exists an extensive data set of elliptic flow measurements in $\mathrm{Au}+\mathrm{Au}$ collisions at RHIC as a function of center-of-mass energy, centrality, pseudorapidity, and transverse momentum [5-12]. The magnitude of the observed flow anisotropy is found to be strongly correlated with the anisotropic shape of the initial nuclear overlap region. This is expected if interactions among the initially produced particles are very strong, leading to anisotropic pressure gradients, which transform the initial spatial eccentricity into a final momentum anisotropy [18].

Quantitatively, the connection between initial spatial and final momentum anisotropy is explored by hydrodynamic calculations that, for a given equation of state, relate a given 
initial source distribution to the final momentum distribution of the produced particles. For $\mathrm{Au}+\mathrm{Au}$ collisions at the top RHIC energy, $\sqrt{s_{\mathrm{NN}}}=200 \mathrm{GeV}$, such calculations are in good agreement with the elliptic flow data at midrapidity $[3,19]$. From similar studies, it has been numerically established that, for not too large impact parameters, the final magnitude of the elliptic flow is proportional to the initial eccentricity $\epsilon$ used to characterize the spatial anisotropy in the transverse plane of the matter created in the overlap region of the colliding nuclei $[15,20]$. More generally, one expects the ratio of elliptic flow and eccentricity, $v_{2} / \epsilon$, at midrapidity to be a universal function of density and size of the system at the time when the elliptic flow develops $\left(v_{2} / \epsilon\right.$ scaling) [21-23]. In hydrodynamics, this function depends parametrically on the speed of sound in the fireball medium [20].

The elliptic flow in $\mathrm{Cu}+\mathrm{Cu}$ collisions at $\mathrm{RHIC}$ was found to be comparatively large, especially for near-central collisions, reaching almost the same magnitude as in $\mathrm{Au}+\mathrm{Au}$ collisions for the same fractional cross section [24,25], and much larger than expected from hydrodynamic models [26]. A quantitatively meaningful comparison of the elliptic flow values measured in $\mathrm{Cu}+\mathrm{Cu}$ and $\mathrm{Au}+\mathrm{Au}$ collisions requires dividing out the difference in the eccentricity of the nuclear overlap zone since, for a given centrality, the average eccentricity depends on the size of the colliding nuclei. For the same size of the overlap zone, similar densities are achieved in the two collision systems [27,28], but the $\mathrm{Cu}+\mathrm{Cu}$ system exhibits a significantly smaller spatial eccentricity. If one scales the measured $v_{2}$ by this eccentricity, using its conventional definition in terms of the spatial deformation of the average transverse distribution of participating nucleons at a given impact parameter, one is led to the paradoxical finding that the smaller $\mathrm{Cu}+\mathrm{Cu}$ system translates the initial spatial deformation more efficiently into a final momentum anisotropy than the larger $\mathrm{Au}+\mathrm{Au}$ system $[27,28]$.

However, this conclusion depends on the definition of the eccentricity used in the scaling procedure. In Ref. [29] it was pointed out that the shape of the nuclear overlap fluctuates from event to event, and in Ref. [30] it is emphasized that the orientation of its major axes relative to the reaction plane (defined by the beam axis and impact parameter vector) fluctuates as well. This is illustrated in Fig. 1. For a given event, the actual distribution of the participant interaction points in the overlap zone can cause the overlap zone to be tilted with respect to the reaction plane. The participant eccentricity [ $\epsilon_{\text {part }}$, Eq. (A6) [30]] takes this into account by using the principal axes of the overlap zone. Because of the fluctuations, the ensemble average of the eventwise participant eccentricity is not identical with the standard eccentricity $\left[\epsilon_{\mathrm{s}}\right.$, Eq. (A2)] of the smooth overlap distribution which is obtained by averaging the participant density in the overlap region with respect to the reaction plane over many events.

Since hydrodynamic collective flow is not a property of the event ensemble, but rather develops independently in each collision event, its driving force is the shape and deformation of the initial distribution of produced matter in each event. To investigate the validity of the hydrodynamically predicted $v_{2} / \epsilon$ scaling one should therefore, in principle, compute the

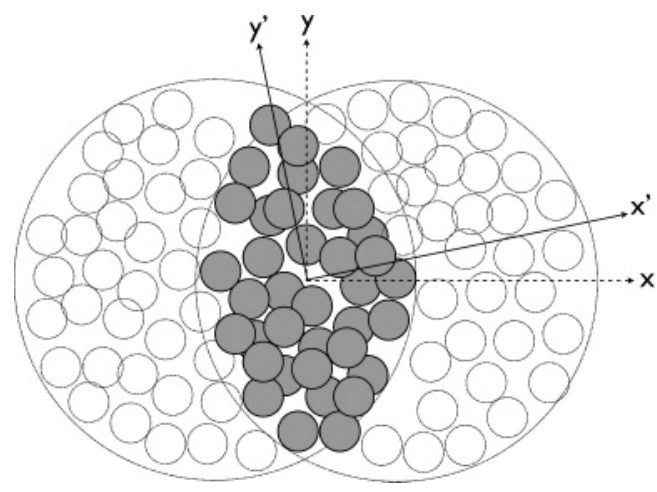

FIG. 1. Schematic illustration of a nucleus-nucleus collision depicted in the transverse plane. The principal axes $\left(x^{\prime}\right.$ and $\left.y^{\prime}\right)$ of the area formed by the participants are tilted with respect to the reaction plane given by the axes ( $x$ and $y$ ) of the transverse plane.

ratio $v_{2} / \epsilon_{\text {part }}$ event by event, before taking its ensemble average $\left\langle v_{2} / \epsilon_{\text {part }}\right\rangle$. This is, unfortunately, not possible in practice since the initial spatial eccentricity $\epsilon_{\text {part }}$ of a given collision event cannot be measured, and a statistically accurate determination of the elliptic flow $v_{2}$ also requires summing the hadron momentum spectra over many events, so only its ensemble average $\left\langle v_{2}\right\rangle$ is known. In practice, the best way to approximate $\left\langle v_{2} / \epsilon_{\text {part }}\right\rangle$ is to scale the measured elliptic flow $\left\langle v_{2}\right\rangle$ by a calculated average participant eccentricity $\left\langle\epsilon_{\text {part }}\right\rangle$ or by a higher moment of it (see below).

In Refs. [24,30] the participant eccentricity scaling was studied using Monte Carlo Glauber (MCG) calculations, where $\epsilon_{\text {part }}$ is computed for each event from the transverse position distribution of nucleons participating in the collision, taken in its individual major axis frame. For large nuclei, eventwise fluctuations in the transverse density distributions are small, except for the most peripheral collisions. Nonetheless, as one approaches zero impact parameter (i.e., in almost central collisions where both $\epsilon_{\mathrm{s}}$ and $\epsilon_{\mathrm{part}}$ are tiny), these small density fluctuations still cause significant relative fluctuations of $\epsilon_{\text {part }}$, resulting in a nonnegligible difference between the participant and the standard eccentricity, even in $\mathrm{Au}+\mathrm{Au}$ collisions. For the smaller $\mathrm{Cu}+\mathrm{Cu}$ system, the eventwise fluctuation effects are much stronger and seriously affect the eccentricity over the entire range of impact parameters [24]. The participant-eccentricity-scaled elliptic flow $\left\langle v_{2}\right\rangle /\left\langle\epsilon_{\text {part }}\right\rangle$ thus differs appreciably from the standard eccentricity-scaled elliptic flow. It appears that scaling $v_{2}$ with the participant eccentricity unifies the eccentricity-scaled elliptic flow $\left\langle v_{2}\right\rangle /\left\langle\epsilon_{\text {part }}\right\rangle$ across the $\mathrm{Cu}+\mathrm{Cu}$ and $\mathrm{Au}+\mathrm{Au}$ collision systems $[24,30]$, even differentially as a function of transverse momentum and pseudorapidity [31]. Furthermore, first measurements of elliptic flow fluctuations have recently been reported in $\mathrm{Au}+\mathrm{Au}$ collisions at $\sqrt{s_{\mathrm{NN}}}=200 \mathrm{GeV}[32,33]$. The relative fluctuation magnitude $\sigma_{\epsilon_{\text {part }}} /\left\langle\epsilon_{\text {part }}\right\rangle$ from MCG is in striking agreement with $\sigma_{v_{2}} /\left\langle v_{2}\right\rangle$ from data [32], as expected if initial state fluctuations are combined with hydrodynamic evolution.

The initial success of the participant eccentricity calculated in the MCG approach immediately suggests a new set of questions: 
(i) How robust are the participant eccentricity results to the parameters characterizing the nuclear density distribution (radius, skin depth, and nucleon-nucleon potential)?

(ii) What is the effect of varying the assumptions about matter production (locality, participant, and binary collision weighting)?

(iii) What features of the MCG initial state distinguish it from the usual optical Glauber model picture?

(iv) More specifically, what is the impact of the fluctuating initial conditions on the suggested [23,29] use of cumulant approaches? In particular, which moment of an underlying fluctuating flow distribution is measured by the (standard) event-plane flow method [17]?

These questions will be addressed in the present paper. In addition, following and improving on Ref. [23], we derive analytical expressions for the eccentricity cumulants in terms of moments of the initial spatial matter distribution, including all leading terms. Furthermore, by comparing with the numerical MCG model, we show that the analytical expressions are misleading, as they neglect important effects arising from spatial correlations between the participating nucleons.

\section{MONTE CARLO GLAUBER MODEL}

To estimate the geometrical configurations of colliding nuclei, one typically constructs models based on rather generic assumptions about the constituent makeup of a typical nucleus. In this context, it is fairly standard to assume that nuclear matter in a nucleus is distributed according to the charge distributions seen in electron scattering experiments. There are two ways of expressing these densities in actual calculations (for an overview, see Ref. [34] and references therein). One way is to assume a smooth matter density, typically described by a Fermi distribution in the radial direction and uniform over solid angle (in the case of spherical or near-spherical nuclei), as done in "optical" Glauber calculations [35,36]. It should be noted that this method neglects some potentially important correlations between participating nucleon positions as will be discussed further in Sec. IV C.

A related, but fundamentally different approach is to distribute, event-by-event in a stochastic manner, individual nucleons according to the smooth matter distribution and to evaluate the collision properties of the colliding nuclei by averaging over multiple events using Monte Carlo methods $[37,38]$. The key ingredients in MCG calculations are the following:

(i) The nucleon position centers in each nucleus are distributed according to a probability distribution given by the smooth nuclear density function $\rho$. One can think of the smooth nuclear density as a quantum mechanical single-particle probability distribution for the nucleon positions and their actual values in an individual collision event as a "measurement" of their positions in a given collision experiment.

(ii) The nucleons are assumed to travel in straight trajectories along the beam direction throughout the reaction, i.e., their transverse positions are "frozen" during the short time when the two high-energy nuclei pass through each other.

(iii) The nucleons interact with nucleons in the oncoming nucleus by means of the nucleon-nucleon inelastic cross section $\left(\sigma_{\mathrm{NN}}\right)$ appropriate for the beam energies under consideration (measured in proton-proton collisions). The nucleon-nucleon collisions occur and produce particles independently, i.e., dynamical correlations among the nucleon positions in the multiparticle nuclear wave function are assumed to be negligible. The only correlations in the model are of geometrical nature and due to clustering effects from the interaction process itself, as explained in Sec. IV C.

Commonly, a nucleon-nucleon collision in the reaction is defined to occur if the Euclidean transverse distance between the centers of any two nucleons is less than the "ball diameter,"

$$
D=\sqrt{\sigma_{\mathrm{NN}} / \pi}
$$

More specifically, the steps of the PHOBOS Monte Carlo [3] calculation for a single event are the following:

(i) Impact parameter selection: The two nuclei are separated in the $x$ direction by an impact parameter $b$ chosen randomly according to $d N / d b \propto b$ up to some large maximum $\left(b_{\max } \simeq 20 \mathrm{fm}>2 R_{A}\right)$. Thus, nucleus $A$ is defined to be centered at $\{x, y\}=\{-b / 2,0\}$ in the transverse plane, while nucleus $B$ is centered at $\{x, y\}=\{+b / 2,0\}$. In addition, both nuclei are centered at $z=0$, since the longitudinal coordinate of each nucleus does not matter for the subsequent steps. ${ }^{1}$

(ii) Makeup of nuclei: For each nucleus, we loop over the number of nucleons, $N_{A}$ and $N_{B}$, and for each nucleon center point choose random, uniformly distributed azimuthal and polar angles, as well as a radius sampled randomly according to the radial density distribution $\rho(r)$. Additionally, to mimic excluded volume effects, one may require a minimum internucleon separation distance $\left(d_{\text {min }}\right)$ between the nucleon centers of all nucleons in the nucleus. This introduces a geometrical correlation among the nucleon positions. In the construction of the nuclei, we make sure that the center of mass of the nuclei is correctly positioned; i.e., we achieve $\sum x_{i}= \pm b / 2, \sum y_{i}=0$, and $\sum z_{i}=0$ by shifting all nucleon centers by the average offset determined after the positions of all nucleons in the nucleus have been generated. Thereby we ensure that the nuclear reaction and the otherwise arbitrary MC frames coincide.

(iii) Collision determination: For each nucleon in nucleus $A$, we loop over the nucleons in nucleus $B$. If the twodimensional Euclidean distance $\sqrt{\Delta x^{2}+\Delta y^{2}}$ between the nucleon from $A$ and the nucleon from $B$ is less than $D$ as defined in Eq. (1), the number of collisions

\footnotetext{
${ }^{1}$ Throughout the paper, we will keep the common choice that the reaction plane, defined by the impact parameter and the beam direction, is given by the $x$ and $z$ axes, while the transverse plane is given by the $x$ and $y$ axes.
} 
TABLE I. Default parameters used in the PHOBOS MCG calculations for $\mathrm{Au}+\mathrm{Au}$ and $\mathrm{Cu}+\mathrm{Cu}$ at $\sqrt{s_{\mathrm{NN}}}=200 \mathrm{GeV}$.

\begin{tabular}{lcrccc}
\hline \hline System & $\sigma_{\mathrm{NN}}(\mathrm{mb})$ & $N_{A / B}$ & $R(\mathrm{fm})$ & $a(\mathrm{fm})$ & $d_{\text {min }}(\mathrm{fm})$ \\
\hline $\mathrm{Au}+\mathrm{Au}$ & 42 & 197 & 6.38 & 0.535 & 0 \\
$\mathrm{Cu}+\mathrm{Cu}$ & 42 & 63 & 4.20 & 0.596 & 0 \\
\hline \hline
\end{tabular}

suffered by both nucleons is incremented by 1 . If no such nucleon-nucleon collision is registered for any pair of nucleons, then no nucleus-nucleus collision occurred. Counters for determination of the total (geometric) cross section are updated accordingly.

Having access to the number of collisions suffered by each nucleon according to this purely geometrical (classical) prescription allows straightforward calculation of $N_{\text {part }}$, the number of nucleons which are struck at least once, and $N_{\text {coll }}$, the total number of nucleon-nucleon collisions. The latter is defined as the sum of collisions suffered by nucleons in one nucleus with nucleons from the other one (to avoid double counting). For every collision, the calculation keeps track of the position and status of each nucleon in the event, for later usage in the calculation of the spatial eccentricity or any other interesting quantity.

The default parameters of the PHOBOS Glauber calculations for $\mathrm{Au}+\mathrm{Au}$ and $\mathrm{Cu}+\mathrm{Cu}$ collisions at $\sqrt{s_{\mathrm{NN}}}=200 \mathrm{GeV}$ are listed in Table I. The nucleon-nucleon inelastic cross section of $\sigma_{\mathrm{NN}}=42 \mathrm{mb}$ is from Ref. [39], while the parameters for the Fermi distribution

$$
\rho(r) \propto\left(1+\exp \left(\frac{r-R}{a}\right)\right)^{-1},
$$

i.e., the nuclear radius $R$ and the skin depth $a$, are from Ref. [40]. The minimum internucleon separation distance is set to $d_{\min }=0 \mathrm{fm}$, i.e., we generally ignore geometrical correlations in the multinucleon wave function due to a hard core, since their effect, especially on the participant eccentricity, is found to be small, as we will report in Sec. III A.

A few comments are in order explaining what this model delivers and how we subsequently interpret its output. As described so far, the MCG model records only the (transverse) position and collision status of each nucleon. No particles are produced in the calculation, and dynamical correlations among the nucleons in the nuclear wave function are neglected. By specifying the nuclear positions exactly, i.e., as long as we do not allow for a smearing around the points given by the model, we are prohibited (by quantum mechanical uncertainty) from imposing any constraints on the momenta of the scattered nucleons and, in consequence, of the particles produced by the collision. Source eccentricities calculated directly from the distribution of (exact) nucleon positions obtained from the MCG model can therefore, at least in principle, not immediately be assumed to represent the eccentricity of the produced matter distribution which drives the anisotropy of the subsequent collective expansion. For this reason, we extend in Sec. III B the model by smearing the resulting nucleon-nucleon collision points with a profile function in order to model the production of (approximately thermalized) matter with finite temperature and restricted particle momenta in the neighborhood of the collision points delivered by the MCG model. The (in-)sensitivity of the source eccentricity of these matter distributions to the parameters of the smearing profile is studied in detail.

\section{ROBUSTNESS OF THE ECCENTRICITY}

In this section, we evaluate the effects of variations in the nuclear density distributions and of various assumptions about the sources and spatial localization of the initial matter distributions on the eccentricity and its centrality dependence. The two definitions of eccentricity considered in this section are the reaction-plane eccentricity [see Eq. (A3)]

$$
\epsilon_{\mathrm{RP}}=\frac{\sigma_{y}^{2}-\sigma_{x}^{2}}{\sigma_{y}^{2}+\sigma_{x}^{2}},
$$

and the participant eccentricity [see Eq. (A6)]

$$
\epsilon_{\mathrm{part}}=\frac{\sqrt{\left(\sigma_{y}^{2}-\sigma_{x}^{2}\right)^{2}+4 \sigma_{x y}^{2}}}{\sigma_{y}^{2}+\sigma_{x}^{2}},
$$

where $\sigma_{x}^{2}, \sigma_{y}^{2}$, and $\sigma_{x y}$ are the (co-)variances of the participantweighted nucleon distribution in a given MCG event. ${ }^{2}$ Their definitions and relation to the standard eccentricity $\left[\epsilon_{\mathrm{s}}\right.$, Eq. (A2)] of the event-averaged distribution, are discussed in Appendix A.

\section{A. Variation of density parameters}

Before Refs. [29,30] were published, the purpose of MCG calculations was to estimate global properties of nucleusnucleus collisions, i.e., to calculate centrality- and eccentricityrelated quantities on average, based on (large) samples of Glauber events. Since both the participant eccentricity and the reaction-plane eccentricity explicitly involve an interpretation of each MCG event individually, it is important to understand their dependence on the choice of the MCG calculation parameters. A number of sources of systematic error are studied by varying a specific parameter with respect to the baseline parameter set as listed in Table II. The baseline values for the sensitivity study correspond to the default parameter set for $\sqrt{s_{\mathrm{NN}}}=200 \mathrm{GeV}$ except for the minimum internucleon separation distance which is here set to $d_{\min }=$ $0.4 \mathrm{fm}$ to match the default value in the HIJING code [41]. We study the variation of all the main Glauber parameters except for the atomic mass number. The nuclear radius $R$ is varied by $\pm 2 \%$, the nuclear skin depth $a$ by $\pm 10 \%$; both variations are several times larger than the systematic error assigned to their measurements by the authors of Ref. [40]. The nucleon-nucleon inelastic cross section $\sigma_{\mathrm{NN}}$ is varied by more than the experimentally spanned region at RHIC. ${ }^{3}$

\footnotetext{
${ }^{2}$ Both definitions have already been used in Refs. [24,30].

${ }^{3} A$ posteriori, this is justified since the dependence on $\sigma_{\mathrm{NN}}$ turns out to be small. Thus, this approach will allow the treatment of the systematics at all collision energies in the same way.
} 
TABLE II. Baseline, minimum and maximum values of parameters in the PHOBOS MCG calculations for $\mathrm{Au}+\mathrm{Au}$ and $\mathrm{Cu}+\mathrm{Cu}$ collisions used in sensitivity studies of the eccentricity definitions.

\begin{tabular}{lccccccc}
\hline \hline Parameter & Base & & $\begin{array}{c}\text { Au+Au } \\
\text { Min }\end{array}$ & Max & Base & Min & Max \\
\hline$\sigma_{\mathrm{NN}}$ & $(\mathrm{mb})$ & 42 & 30 & 45 & 42 & 30 & 45 \\
$R$ & $(\mathrm{fm})$ & 6.38 & 6.25 & 6.51 & 4.22 & 4.14 & 4.30 \\
$a$ & $(\mathrm{fm})$ & 0.535 & 0.482 & 0.586 & 0.596 & 0.536 & 0.656 \\
$d_{\min }$ & $(\mathrm{fm})$ & 0.4 & 0 & 0.8 & 0.4 & 0 & 0.8 \\
\hline \hline
\end{tabular}

The minimum internucleon separation distance, which is not known experimentally, is varied by $\pm 100 \%$. As one can see in Fig. 2, both eccentricity definitions are quite stable within the studied range of Glauber parameters. In particular, this is true for the participant eccentricity. Not unexpectedly, the difference between the two definitions, $\epsilon_{\text {part }}$ and $\epsilon_{\mathrm{RP}}$, is most pronounced for $\mathrm{Cu}+\mathrm{Cu}$. We have also found that varying two parameters at the same time does not increase significantly the observed variation in eccentricity.

\section{B. Particle production models}

\section{Binary collisions vs participants}

The observed particle multiplicity at midrapidity scales somewhat more strongly than linearly with the number of participating nucleons [42]. This can be parametrized by postulating a second (smaller) contribution to particle production that scales with the number of binary nucleonnucleon collisions [43]. The MCG model as described above, can be extended to implement matter distributions produced according to a "two-component" scenario, where some of the matter is generated proportionally to the number of binary collisions. To add this feature, it is necessary to define two origins of matter production: (1) participant nucleons, which create the matter by means of "exciting" the nucleon, and (2) binary nucleon-nucleon collisions, which create the matter locally via a two-body interaction.

The latter mechanism suggests that the produced matter, to be incorporated into the calculation of spatial eccentricity, should be centered between the colliding nucleons. We achieve
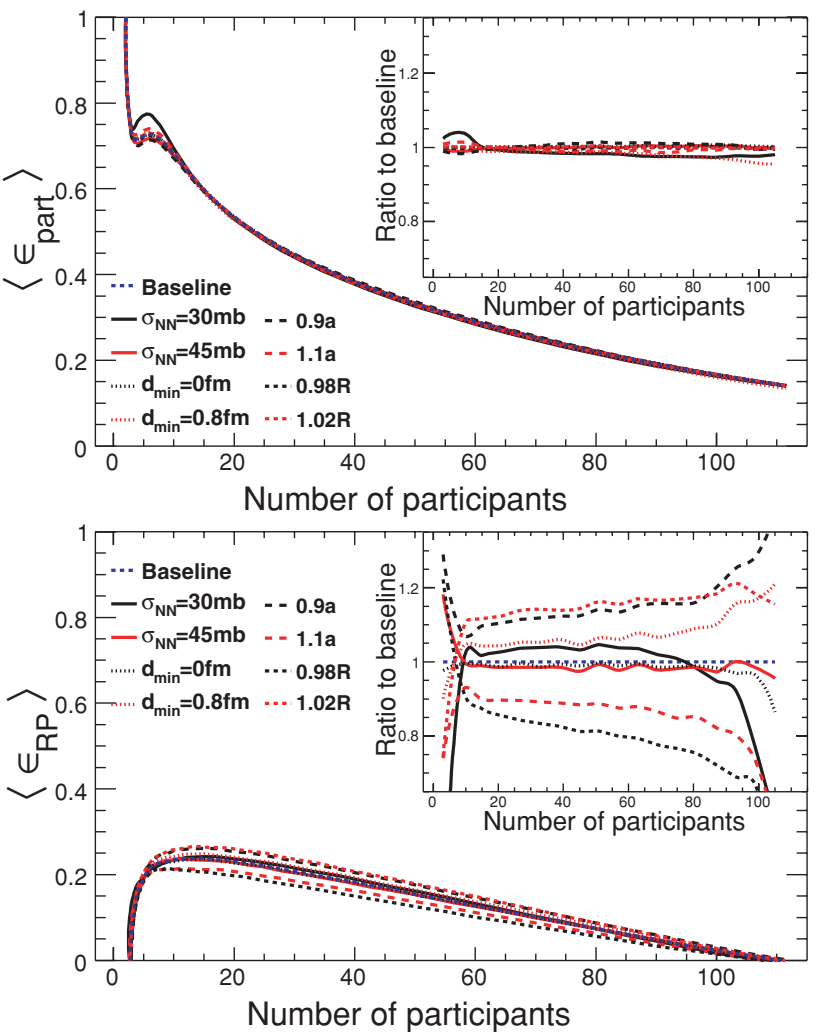

FIG. 2. (Color online) Participant (top) and reaction-plane (bottom) eccentricity in $\mathrm{Au}+\mathrm{Au}$ (left) and $\mathrm{Cu}+\mathrm{Cu}$ (right) collisions as a function of $N_{\text {part }}$ for variations of the Glauber parameters as given in Table II. The inset shows the ratio with respect to the baseline calculation in each case. 


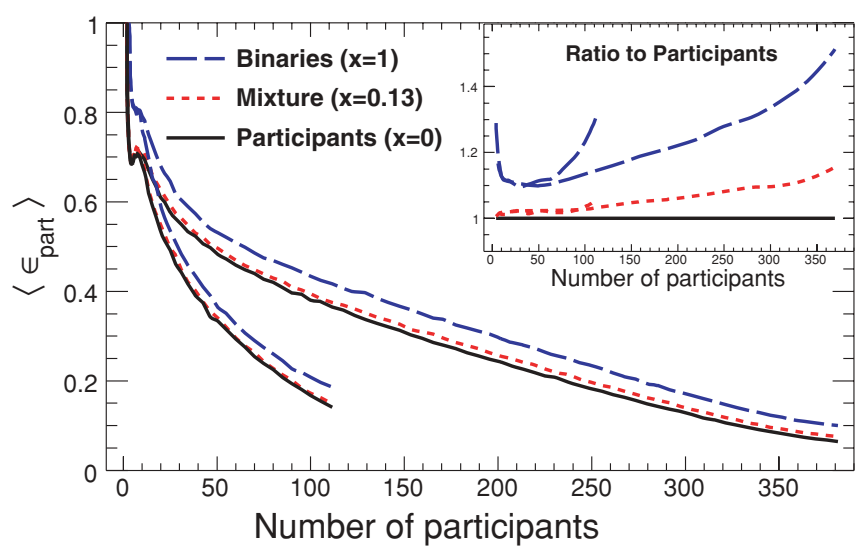

FIG. 3. (Color online) Comparison of participant vs binary weighted participant eccentricity, as well as the $x=0.13$ mixture, as a function of $N_{\text {part }}$ in $\mathrm{Au}+\mathrm{Au}$ (upper set of curves) and $\mathrm{Cu}+\mathrm{Cu}$ (lower set of curves) collisions at $\sqrt{s_{\mathrm{NN}}}=200 \mathrm{GeV}$. The inset shows the ratio with respect to the participant-weighted eccentricity.

this by means of a "pseudoparticle" that is located at the center of mass of the pair of colliding nucleons and keeps track of the "collision-weighted" matter with an appropriate weight $x$. Thus, contributions to the eccentricity from participants are weighted by $(1-x) / 2$, while those from binary collisions by $x$. In Fig. 3, the results for purely participant $(x=0)$ and purely collision $(x=1)$ weighted participant eccentricity are shown and compared with the case $x=0.13$ which has been found to describe the centrality dependence of the multiplicity at midrapidity according to $d N_{\mathrm{ch}} / d \eta=d N_{\mathrm{ch}}^{\mathrm{pp}} / d \eta\left[\frac{1-x}{2} N_{\text {part }}+\right.$ $x N_{\text {coll }}$ [ [42]. Independent of centrality, the collision-weighted $\epsilon_{\text {part }}$ values are shifted to larger eccentricity for all centralities, similar to what is known for the standard eccentricity [44]. The presumably realistic case of $x=0.13$ yields about $10 \%$ larger eccentricity in the most central $\mathrm{Au}+\mathrm{Au}$ collisions.

\section{Effects of smeared matter distributions}

The driving force for hydrodynamic elliptic flow is not directly the eccentricity of the distribution of participating nucleons or binary nucleon-nucleon collisions, but rather the anisotropy of the pressure gradients in the initially produced hot matter after it has thermalized. The transverse distribution of this matter will be smeared around the transverse positions of the participating nucleons or binary collision points. To study the behavior of the eccentricity under different definitions, we introduce a general procedure for incorporating a variety of matter density distributions. In this approach, the contribution of each matter production point (i.e., the center of a participant nucleon or a binary collision) at $\left(x_{i}, y_{i}, z_{i}\right)$ is smeared according to $P\left(x-x_{i}, y-y_{i}, z-z_{i}\right)$, leading to a continuous weight function defined at all space-points in the transverse plane $w(x, y, z)=\sum P\left(x-x_{i}, y-y_{i}, z-\right.$ $\left.z_{i}\right)$. Averages and higher moments in space-time are then calculated (for individual events) using this weight function, e.g., $\{x\}=\int x w(x, y, z) d x d y d z$. The pointlike MCG cases described above correspond to the choice of $P\left(x-x_{i}, y-\right.$

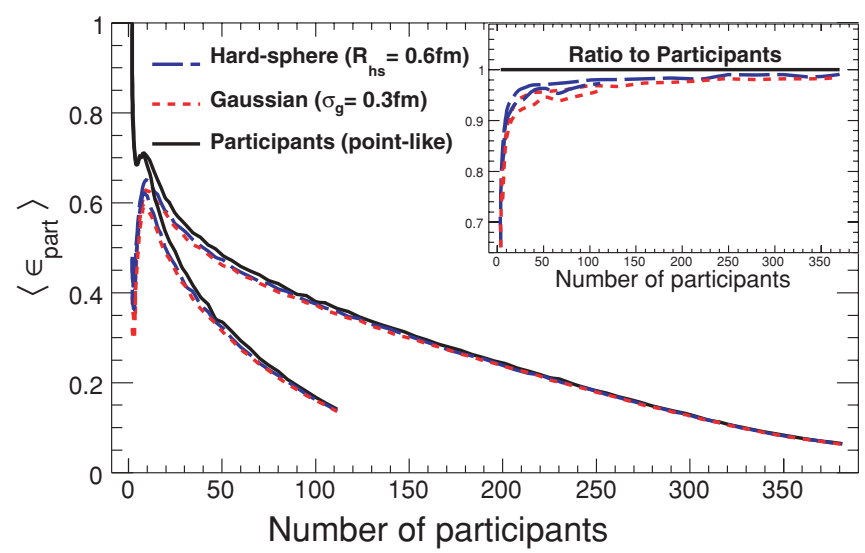

FIG. 4. (Color online) Comparison of participant eccentricity as a function of $N_{\text {part }}$ in $\mathrm{Au}+\mathrm{Au}$ (upper set of curves) and $\mathrm{Cu}+\mathrm{Cu}$ (lower set of curves) collisions at $\sqrt{s_{\mathrm{NN}}}=200 \mathrm{GeV}$ for pointlike, hard-sphere, and Gaussian matter distributions. The inset shows the ratio with respect to the pointlike participant eccentricity.

$\left.y_{i}, z-z_{i}\right)=\delta\left(x-x_{i}\right) \delta\left(y-y_{i}\right) \delta\left(z-z_{i}\right)$. We look at two different, azimuthally symmetric, parametrizations for the smearing profile:

(i) Hard-sphere smearing, $P_{\mathrm{hs}}(r) \propto r^{2} \theta\left(R_{\mathrm{hs}}^{2}-r^{2}\right)$,

(ii) Gaussian smearing, $P_{\mathrm{g}}(r) \propto r^{2} \exp \left(-r^{2} / 2 \sigma_{\mathrm{g}}^{2}\right)$,

where $r^{2}=\left(x-x_{i}\right)^{2}+\left(y-y_{i}\right)^{2}+\left(z-z_{i}\right)^{2}$, and $\theta$ denotes the step function. In the following, we estimate meaningful choices for the parameters, $R_{\mathrm{hs}}$ and $\sigma_{\mathrm{g}}$. For $R_{\mathrm{hs}}$, it makes sense to use the interaction radius, $R_{\mathrm{hs}}=D / 2=\sqrt{\sigma_{\mathrm{NN}} / 4 \pi}$, since the nucleons in the Glauber model are assumed to interact if their centers are within the ball diameter $D$, Eq. (1). For $200 \mathrm{GeV}$, this corresponds to $R_{\mathrm{hs}} \approx 0.6 \mathrm{fm}$. Then, matching the rms width of the Gaussian distribution to the rms width of the hard sphere, $\sigma_{\mathrm{g}}^{2}=R_{\mathrm{hs}}^{2} / 5$, leads to $\sigma_{\mathrm{g}} \approx 0.3 \mathrm{fm}$. In Fig. 4 , we show the results for the participant eccentricity calculated for pointlike, hard-sphere, and Gaussian local matter distributions, using this set of parameters. The comparison reveals that for both collision systems, the way the produced matter is distributed around the MCG interaction points does not significantly influence the observed value of the eccentricity except for extremely small systems. This also shows that the quantum mechanical uncertainty on the transverse positions of the interaction points has no major influence on the initial source eccentricity. Note that similarly to what was reported in Ref. [45] we find significantly different centrality dependence for $\epsilon_{\text {part }}$ if we allow smearing out the local matter sources to a very large extent. For example, $\epsilon_{\text {part }}$ in $\mathrm{Au}+\mathrm{Au}$ collisions at all centralities does not exceed 0.15 for Gaussian smearing with $\sigma_{\mathrm{g}}=2 \mathrm{fm}$.

\section{CORRELATIONS AND FLUCTUATIONS}

In this section, we focus on eccentricity cumulants (which enter the discussion and interpretation of elliptic flow data) in the context of fluctuating initial conditions and for different realizations of the Glauber model initial state. 


\section{A. Sensitivity of the event-plane method to underlying flow fluctuations}

There are different ways of extracting the elliptic flow from data: the event-plane method, two-particle correlations, multiparticle cumulants, etc. (see Refs. $[17,46]$ ). Each flow measurement is based on a different moment of the finalparticle momentum distribution and thus is differently affected by event-by-event flow fluctuations (and nonflow correlations). If elliptic flow is proportional to the spatial anisotropy, the eccentricity scaling should be performed with corresponding moments of the participant eccentricity [23,29].

It has been explicitly stated [23] (see also Ref. [46]) that the event-plane method $\left(v_{2}\{\mathrm{EP}\}\right)$, used by the PHOBOS experiment to measure elliptic flow, really measures $\sqrt{\left\langle v_{2}^{2}\right\rangle}$ rather than $\left\langle v_{2}\right\rangle$, i.e., the rms rather than the mean of $v_{2}$. More specifically, it has been claimed that $\left\langle v_{2}\right\rangle \leqslant v_{2}\{\mathrm{EP}\} \leqslant \sqrt{\left\langle v_{2}^{2}\right\rangle}$ depending on the event-plane resolution, with the upper limit being approximately reached under RHIC conditions [47]. Here, $\langle\ldots\rangle$ indicates an average over many collision events. In the following, we will investigate and confirm this claim.

In the event-plane method [17], one uses the particles from one side of the detector (subevent $A$ ) to estimate the event plane, the plane relative to which the flow develops, given by $\Psi_{2}^{A}=\frac{1}{2} \tan ^{-1}\left[\sum \sin \left(2 \phi_{i}\right) / \sum \cos \left(2 \phi_{i}\right)\right]$. One then correlates the particles from the other, symmetric, side of the detector (subevent $B$ ) with this event plane to obtain the uncorrected flow signal for the given subevent, $v_{2, B}^{\mathrm{obs}}=\left\{\cos \left(2 \phi_{i}-2 \Psi_{2}^{A}\right)\right\}$. Here, as before and in the appendixes, $\{\ldots\}$ indicates the average over an individual event. The roles of subevents $A$ and $B$ can then be interchanged to obtain $\Psi_{2}^{B}, v_{2, A}^{\text {obs }}$, and thus the observed flow signal for the whole event $v_{2}^{\text {obs }}$. Assuming small dynamical and nonflow correlations, one has (for symmetric subevents)

$$
\left\langle v_{2, B(A)}^{\text {obs }}\right\rangle=\left\langle v_{2}\right\rangle\left\langle\cos \left(2 \Psi_{2}-2 \Psi_{2}^{A(B)}\right)\right\rangle,
$$

where the average is, unlike before, not over particles in a given event but over events in the given centrality, $\eta$ and $p_{\mathrm{T}}$ bin. In this equation, $\Psi_{2}$ stands for the actual event plane angle, which defines the orientation of the $v_{2}$ signal in a particular event [24], and $\left\langle\cos \left(2 \Psi_{2}-2 \Psi_{2}^{A B}\right)\right\rangle \equiv R$ quantifies the event-plane resolution, which itself depends on $v_{2}$ [17]. The resolution can be estimated based on data alone,

$$
R=\sqrt{\left\langle\cos \left(2 \Psi_{A}-2 \Psi_{B}\right)\right\rangle},
$$

leading to $\left\langle v_{2}^{\text {obs }}\right\rangle=\left\langle v_{2}\right\rangle R$. The presence of a fraction $f_{\text {bkg }}$ of uncorrelated background particles in addition to the $N$ particles that carry the flow signal corresponds (restricting ourselves to second harmonic contributions) to a distribution of

$$
\frac{d N}{d \phi}=\left(1+f_{\mathrm{bkg}}\right) \frac{N}{2 \pi}\left[1+\frac{2 v_{2}}{1+f_{\mathrm{bkg}}} \cos \left(2 \phi-2 \Psi_{2}\right)\right],
$$

leading to an apparent suppression of the observed flow signal by $1+f_{\text {bkg }}$. Correcting for this effect, we arrive at the final expression for the flow measured via the event-plane method

$$
v_{2}\{\mathrm{EP}\} \equiv\left\langle v_{2}\right\rangle=\frac{1+f_{\text {bkg }}}{R}\left\langle v_{2}^{\text {obs }}\right\rangle .
$$

This includes the two main experimental corrections, namely, for suppression and event-plane resolution [8].

From Eq. (5) it is not obvious if the $v_{2}\{\mathrm{EP}\}$ scales with the mean or the rms of an underlying $v_{2}$ distribution and how such a behavior depends on $R$. However, one can study this behavior numerically with a MC calculation that creates events with $v_{2}$ distributed according to a given distribution $P\left(v_{2}\right)$. For every event, we take $N$ particles (at midrapidity) to carry the flow signal, according to $d N / d \phi \propto 1+2 v_{2} \cos (2 \phi)$, and $f_{\text {bkg }} N$ particles $\left(f_{\text {bkg }} \geqslant 0\right)$ to represent the uncorrelated background, which are added with a uniform azimuthal distribution. To relate the obtained $v_{2}\{\mathrm{EP}\}$ to a moment of the input distribution, we implicitly define the exponent $\alpha$ according to

$$
\left\langle v_{2}^{\alpha}\right\rangle^{\frac{1}{\alpha}}=v_{2}\{\mathrm{EP}\},
$$

where the ensemble average $\langle\langle\cdots\rangle\rangle$ is calculated with the underlying $v_{2}$ distribution $P\left(v_{2}\right)$. One obtains the sensitivity of the event-plane method to $P\left(v_{2}\right): \alpha=1$ corresponds to a scaling of $v_{2}\{\mathrm{EP}\}$ with the mean, $\alpha=2$ to a scaling with the rms of $P\left(v_{2}\right)$, also often denoted $v_{2}\{2\}$ [46]. In our calculation, we choose $v_{2}$ to be uniformly distributed between $v_{2, \min }$ and $v_{2, \max }$; i.e., the true mean and rms are $0.5\left(v_{2, \min }+v_{2, \max }\right)$ and $\sqrt{\left(v_{2, \min }^{2}+v_{2, \min } v_{2, \max }+v_{2, \max }^{2}\right) / 3}$, respectively.

Figure 5 shows the result of the calculation for various combinations of $v_{2, \min }$ and $v_{2, \max }$ between 0.01 and 0.3 , various values of $N$ between 30 and 1000, as well as values of $f_{\mathrm{bkg}}$ between 0 and 5. For each set of parameters, $10^{7}$ events have been simulated. The number of particles in each event is not allowed to fluctuate, i.e., exactly $N+f_{\text {bkg }} N$ particles are created in every event. Within a reasonable spread that increases with decreasing resolution, the $\alpha$ values are found to lie on a common curve as a function of $R$, with no or weak dependence at most on the chosen simulation parameters. The PHOBOS $v_{2}\{\mathrm{EP}\}$ measurements lie in the range of $0.15 \lesssim R \lesssim 0.55$ for $\mathrm{Au}+\mathrm{Au}$ and $0.13 \lesssim R \lesssim 0.33$ for $\mathrm{Cu}+\mathrm{Cu}$, where $v_{2}\{\mathrm{EP}\}$ scales approximately with the rms

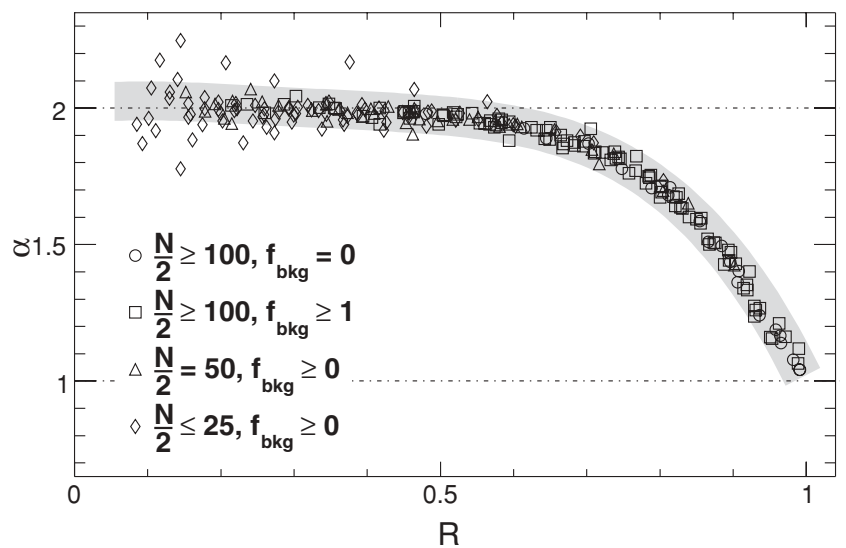

FIG. 5. Dependence of $\alpha$, Eq. (6), on the event-plane resolution $R$ for $P\left(v_{2}\right)$ uniform with various combinations of $v_{2, \min }$ and $v_{2, \max }$, number of signal $N$ and background $f_{\mathrm{bkg}} N$ particles in the MC calculation (see text for more details). The shaded band covers the parameter errors obtained from a polynomial fit to the data. 
of the underlying $v_{2}$ distribution. This result is supported by recent full detector simulations with HIJING MC events that incorporate known, but fluctuating, $v_{2}$ values. For the measured value of $\sigma_{v_{2}} /\left\langle v_{2}\right\rangle \approx 40 \%$ [32] this implies that the PHOBOS $v_{2}\{\mathrm{EP}\}$ measurements are about $10 \%$ larger than the mean elliptic flow.

\section{B. Participant-eccentricity-scaled elliptic flow}

It is expected [21,22] that $v_{2} / \epsilon$ scales with the transverse charged particle area density at midrapidity according to $v_{2} / \epsilon \propto 1 / S d N_{\mathrm{ch}} / d y$, where $S$ is the overlap area in the transverse plane. The data are measured in bins of centrality, translating into

$$
\left.\frac{v_{2}\{\mathrm{EP}\}}{\epsilon\{\mathrm{EP}\}} \propto \frac{1}{\langle S\rangle}\left\langle d N_{\mathrm{ch}} / d y\right\rangle\right|_{|y| \leqslant 1} .
$$

Here, $v_{2}\{\mathrm{EP}\}$ is the PHOBOS estimate of the ensembleaveraged elliptic flow according to Eq. (5), and $\epsilon\{\mathrm{EP}\}$ is a suitable ensemble-averaged initial source eccentricity. In view of the discussion of the preceding subsection, $v_{2}\{\mathrm{EP}\}$ is about $v_{2}\{2\}$, the rms of the underlying $v_{2}$ distribution. Following the suggestion of Refs. [23,29], we therefore scale it with $\epsilon\{\mathrm{EP}\}=\epsilon_{\text {part }}\{2\} \equiv \sqrt{\left\langle\epsilon_{\text {part }}^{2}\right\rangle}$, the rms of the participant eccentricity distribution obtained from the MCG model. The overlap area

$$
S=\pi \sqrt{\sigma_{x}^{2} \sigma_{y}^{2}-\sigma_{x y}^{2}}
$$

corresponds to the area of the tilted overlap ellipse [see Appendix A, and especially Eq. (A8)].

To arrive at the centrality average for $\epsilon_{\text {part }}\{2\}$ and $S$, we fold their $N_{\text {part }}$ dependence with the distribution of $N_{\text {part }}$ values obtained for each centrality bin from full detector simulations. Independent of species and energy, we scale the elliptic flow data by 0.9 and the midrapidity yields by 1.15 to convert from pseudorapidity $\eta$ to rapidity $y$ as described in Refs. [10,48]. Figure 6 shows the results for $\mathrm{Cu}+\mathrm{Cu}$ and $\mathrm{Au}+\mathrm{Au}$ collisions at 62.4 and $200 \mathrm{GeV}$. The flow data are from Refs. [9,24], and the midrapidity yields from Refs. [49-51]. We distinguish two types of errors that are individually propagated in the error calculation of the ratios: (1) systematic and statistical errors (if available) from data added in quadrature to obtain total $90 \%$ C.L., and (2) systematic errors (90\% C.L.) assigned to the MC quantities obtained by the variation of Glauber parameters with respect to the individual baseline values (cf. Table II). As reported earlier [24,30], we find a common scaling between the different systems. However, within the errors, it is difficult to tell whether the almost linear rise of the eccentricity-scaled elliptic flow breaks down at larger values of the area density which might indicate that the hydrodynamic limit is being reached at the top RHIC energy.

\section{Cumulants and correlations}

As mentioned above, it is suggested [29] that higher order cumulant moments of $v_{2}$ should be proportional to analogously defined higher order cumulant moments of the eccentricity,

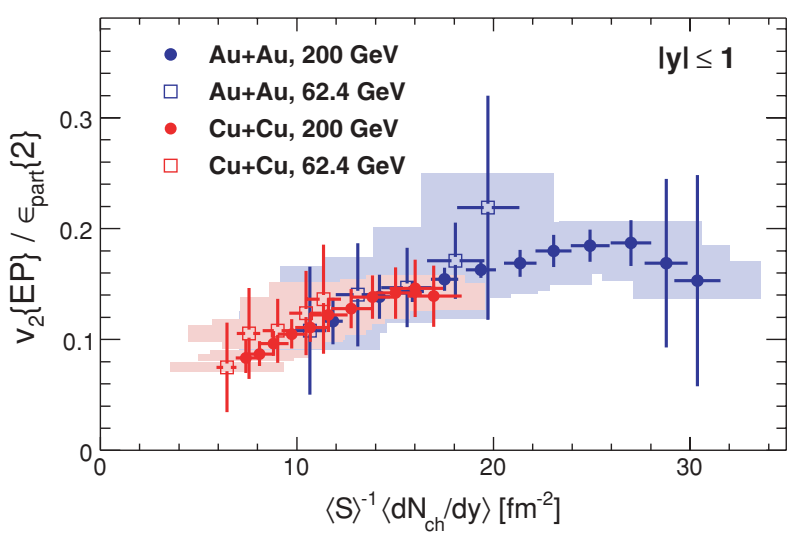

FIG. 6. (Color online) Participant-eccentricity-scaled elliptic flow vs transverse charged particle area density at midrapidity for $\mathrm{Cu}+\mathrm{Cu}$ and $\mathrm{Au}+\mathrm{Au}$ collisions at $\sqrt{s_{\mathrm{NN}}}=62.4$ and $\sqrt{s_{\mathrm{NN}}}=$ $200 \mathrm{GeV}$. The horizontal and vertical error bars originate from the combined statistical and systematic errors of the data (90\% C.L.). The shaded boxes are the result of the systematic errors assigned to $\epsilon_{\text {part }}\{2\}$ and $S$ by the variation of the MCG parameters (90\% C.L.).

including

$$
\begin{aligned}
& \epsilon\{2\}^{2} \equiv\left\langle\epsilon^{2}\right\rangle, \\
& \epsilon\{4\}^{4} \equiv 2\left\langle\epsilon^{2}\right\rangle^{2}-\left\langle\epsilon^{4}\right\rangle .
\end{aligned}
$$

In Ref. [23], Bhalerao and Ollitrault (B\&O) attempted to derive expressions for $\epsilon_{\text {part }}\{2\}$ and $\epsilon_{\text {part }}\{4\}$ semianalytically, making use of two strong approximations. First, the paper contains an implicit assumption that all of the participant positions are independent samples of some underlying distribution, or at least that any correlations between participants do not affect the eccentricity fluctuations [47]. Second, the expressions in Ref. [23] were obtained using a Taylor expansion, leading to a power series in $1 / N_{\text {part }}$ which is then truncated at $1 / N_{\text {part }}$. Based on these approximations, they concluded that $\epsilon_{\text {part }}\{4\}$ is numerically equal to the standard eccentricity $\epsilon_{\mathrm{s}}$, vanishing for central $(b=0)$ collisions. This would in turn imply that higher order cumulants of the flow such as $v_{2}\{4\}$ are insensitive to fluctuations in the participant distribution. In this section, we show that B\&O's assumptions are too strong and that $\epsilon_{\text {part }}\{4\}$ for $\mathrm{Cu}+\mathrm{Cu}$ collisions differs significantly from $\epsilon_{\mathrm{s}}$ when better approximations are made, especially when the role of correlations is taken into account, e.g., for the usual PHOBOS MCG calculations.

\section{Correlations of nucleons in the initial state}

The first approximation in Ref. [23] is to ignore the correlations between nucleon participant positions, even though we know that there are at least three sources for such correlations.

First, to contribute to the produced matter, the participating nucleons must hit each other, which causes a correlation. For instance, in the case of a peripheral collision with two or three participants, the overlap region of the nuclei may be something like $3 \times 1 \mathrm{fm}$, but the nucleons will necessarily all be within about $1 \mathrm{fm}$ of each other or else they would not be participants. In general, the participant positions will tend to 
(a)

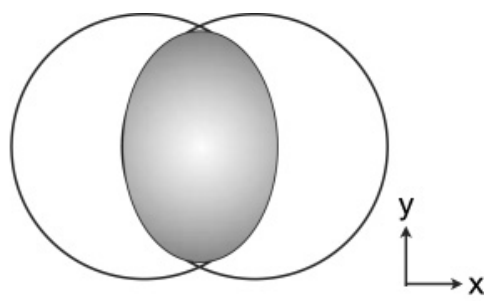

(b)

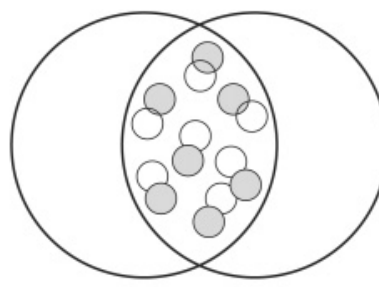

(c)

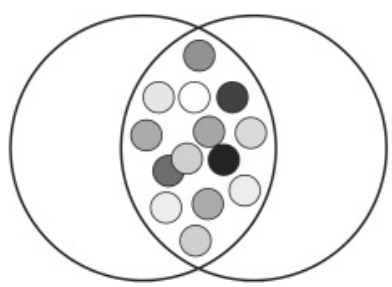

FIG. 7. Schematic of densities for the different approaches: (a) optical limit, (b) full MCG with correlated participants originating from each of the two nuclei in one MCG event, and (c) mixedevent MCG with uncorrelated participants where every participant originates from an individual nucleon-nucleon collision obtained in a different MCG event.

be more clustered in position space than a random distribution, since each participating nucleon must hit another one.

Second, if there are two nucleons from a given nucleus which are close together in transverse position, then they will have a tendency to be either both hit or neither hit. Again this will contribute to clustering of participant positions.

The usual, full PHOBOS MCG calculation takes both of these effects into account automatically, but the analytical expressions, given in Ref. [23] and in Appendix B here, do not. The two different approximations are illustrated in Fig. 7 , diagrams (a) and (b).

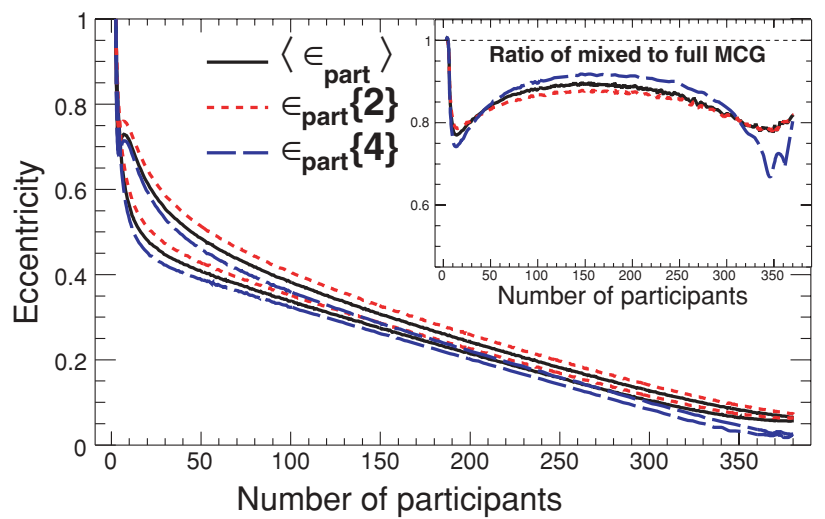

It should be noted that a precise integral Glauber calculation at fixed impact parameter should involve a complete two $(A+B)$-dimensional integral covering all possible transverse positions of all nucleons involved in the collision [34,52,53]. Such a formulation would include the same correlations that are covered automatically by a MCG. The usual practice of approximating the Glauber integral with a single twodimensional integral (optical Glauber model approximation) is just an approximation which neglects all the correlations described above.

A third type of correlation would be genuine nucleonnucleon position correlations in the wave functions of the nuclei that, as mentioned before, generally are ignored in all Glauber model calculations.

\section{Uncorrelated Glauber Monte Carlo}

To estimate the role of pairwise spatial correlations among the participant nucleons in a nucleus-nucleus collision, we make use of a modified version of the MCG approach. In the modified version, at first a normal event is calculated of which we record the impact parameter and corresponding number of participants. Then, a mixed event is constructed from $N_{\text {part }}$ independently calculated events, which are all required to have the same global characteristics, i.e., the same impact parameter and number of participants. From each such event, we choose one of the participating nucleons, such that in the constructed mixed event none of the participating nucleons is correlated to any of the other participants. The resulting approximation of the overlap density is illustrated in diagram (c) of Fig. 7. Figure 8 shows the comparison of the full PHOBOS MCG calculation with the mixed-event MCG calculation for the participant eccentricity and its first two cumulants. We find that the contribution of correlations to $\epsilon_{\text {part }}, \epsilon_{\text {part }}\{2\}$, and $\epsilon_{\text {part }}\{4\}$ is quite important for the smaller system, i.e., it is about $20-45 \%$ for $\mathrm{Cu}+\mathrm{Cu}$ and about $5-10 \%$ for $\mathrm{Au}+\mathrm{Au}$ and rather constant over a wide range of centrality. Note that the structure seen in Fig. 8 at very low $N_{\text {part }}$ values (see also Figs. 2-4) is genuine for the full MCG and not present in the mixed-event case. Furthermore, spatial correlations among the participants in the initial state modeled by MCG are found to be less important

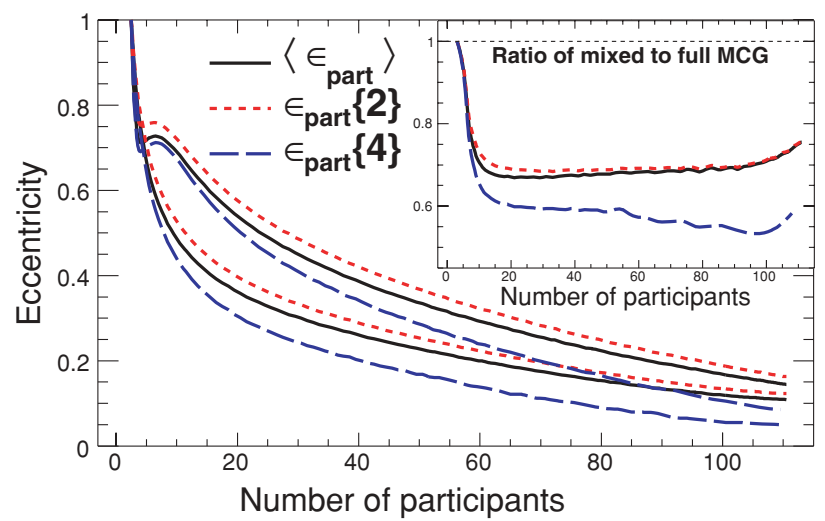

FIG. 8. (Color online) Participant eccentricity and cumulants, $\epsilon_{\text {part }}, \epsilon_{\text {part }}\{2\}$, and $\epsilon_{\text {part }}\{4\}$, as a function of $N_{\text {part }}$ in $\mathrm{Au}+\mathrm{Au}(\mathrm{left})$ and $\mathrm{Cu}+\mathrm{Cu}$ (right) collisions at $\sqrt{s_{\mathrm{NN}}}=200 \mathrm{GeV}$ for the full (upper set of curves) and mixed-event (lower set of curves) MCG calculations. The inset shows the ratio of mixed-event to the full MCG results. 
for the reaction-plane and standard eccentricity definitions (not shown). For both definitions, the uncorrelated cases lead to slightly larger eccentricities. The deviation between full and mixed-event MCG decreases with increasing centrality and is less than a few percent for the $\mathrm{Au}+\mathrm{Au}$ and about 5-15\% for the $\mathrm{Cu}+\mathrm{Cu}$ system.

\section{Cumulants in the extended $B \& O$ approach}

The second approximation in Ref. [23] is to derive expressions for $\epsilon_{\text {part }}\{2\}$ and $\epsilon_{\text {part }}\{4\}$ using a Taylor expansion that leads to a power series in $1 / N_{\text {part }}$. In Appendix B, we advance the calculations to higher orders in $1 / N_{\text {part }}$ by generalizing Eq. (11) from Ref. [23], and we show how to obtain the analytical terms in a rigorous fashion. For $\epsilon_{\text {part }}\{2\}$, we obtain the same expression as Eq. (12) in Ref. [23], and prove that the $\mathcal{O}\left(1 / N_{\text {part }}^{2}\right)$ terms are really negligible. We also obtain the expansion for $\epsilon_{\text {part }}\{4\}$, Eq. (B37), where-in contrast to Ref. [23] - all important terms have been kept. In particular, for central collisions, when $\epsilon_{\mathrm{s}} \rightarrow 0$, some terms of $\mathcal{O}\left(1 / N_{\text {part }}^{3}\right)$ are not negligible and must be kept.

The values for the ensemble averages over participant nucleon distributions (such as $\left\langle r^{2}\right\rangle$ or $\left\langle r^{4} \cos 2 \phi\right\rangle$ ) in Eq. (B37) need to be calculated numerically. We calculate each of these averages as a function of $N_{\text {part }}$ using the usual (full) PHOBOS MCG calculation. Inserting the numerically evaluated values into Eq. (B37) leads to the "semianalytic" result discussed below. Figure 9 shows the results for $\epsilon_{\text {part }}\{4\}$, comparing the full PHOBOS and mixed-event MCG with our semianalytical result from Eq. (B37) and with B\&O's semianalytical approximation [Eq. (13) from Ref. [23] evaluated with the full PHOBOS MCG], as well as with the standard eccentricity $\epsilon_{\mathrm{s}}$. For both collision systems, our semianalytical result fully agrees with the mixed-event MCG calculation. This is consistent with the fact that correlations among the participants are neglected in the analytical derivation of Eq. (B37). Furthermore, it confirms that all numerically important terms have been kept in Eq. (B37). The full MCG calculation which includes participant spatial correlations disagrees with the other calculations that neglect them by almost a factor of 2. Contrary to Ref. [23], we find that, for the $\mathrm{Cu}+\mathrm{Cu}$ system, $\epsilon_{\text {part }}\{4\}$ calculated in the semianalytical approach does not agree with $\epsilon_{\mathrm{s}}$, in particular for very peripheral and near-central collisions. More importantly, however, is the aforementioned effect of the neglected correlations. For the $\mathrm{Au}+\mathrm{Au}$ system, $\epsilon_{\mathrm{part}}\{4\}$ is found to be numerically close to $\epsilon_{\mathrm{s}}$ (with deviations of less than $10 \%$ ) over a wide range of centralities. Only for very peripheral and near-central collisions correlations may play an important role. For the $\mathrm{Cu}+\mathrm{Cu}$ system, on the other hand, $\epsilon_{\text {part }}\{4\}$ differs from $\epsilon_{\mathrm{s}}$ by almost a factor of 2 over a wide range of centralities, implying that correlations cannot be neglected for the smaller system.

Figure 10 shows a comparison of all eccentricity definitions used in the present paper (participant eccentricity and cumulants, as well as reaction-plane and standard eccentricities), obtained from full MCG calculations with baseline parameters listed in Table I.

As mentioned before, in contrast to the results shown in Fig. 9, the results of Ref. [20] indicate that $\epsilon_{\text {part }}\{4\}$ differs very little from both the standard eccentricity $\epsilon_{\mathrm{s}}$ and the average reaction-plane eccentricity $\left\langle\epsilon_{\mathrm{RP}}\right\rangle$, the latter two being almost equal. Recently, the authors of Ref. [54] have shown that, within a Gaussian model of the event-by-event eccentricity fluctuations, the identity of $\epsilon_{\mathrm{part}}\{4\}$ with $\left\langle\epsilon_{\mathrm{RP}}\right\rangle$ is exact [see Eq. (9) in Ref. [54]] as long as the Gaussian widths for $\epsilon_{\mathrm{RP}}$ and for the correlation term $\rho_{x y} \equiv 2 \sigma_{x y} /\left(\sigma_{x}^{2}+\sigma_{y}^{2}\right)$ are equal. We were able to trace the inequality between $\epsilon_{\text {part }}\{4\}$ and $\left\langle\epsilon_{\mathrm{RP}}\right\rangle$ present in our MCG model (see Fig. 10) to a breakdown of the Gaussian model assumptions made in Ref. [54], in particular for peripheral collisions and small collision systems. We find that in the MCG model, the event-by-event fluctuations of the correlation term $\rho_{x y}$ are indeed Gaussian for midcentral to central $\mathrm{Au}+\mathrm{Au}$ and most central $\mathrm{Cu}+\mathrm{Cu}$ collisions. On the other hand, the event-by-event fluctuations of $\epsilon_{\mathrm{RP}}$ are not well described by a Gaussian function for all $\mathrm{Au}+\mathrm{Au}$ and $\mathrm{Cu}+\mathrm{Cu}$ collisions except the most central ones. Furthermore, for semiperipheral and peripheral collisions, the width of the $\epsilon_{\mathrm{RP}}$ distribution does not agree with the width of $\rho_{x y}$. Consequently, for all but the most central collisions, our MCG
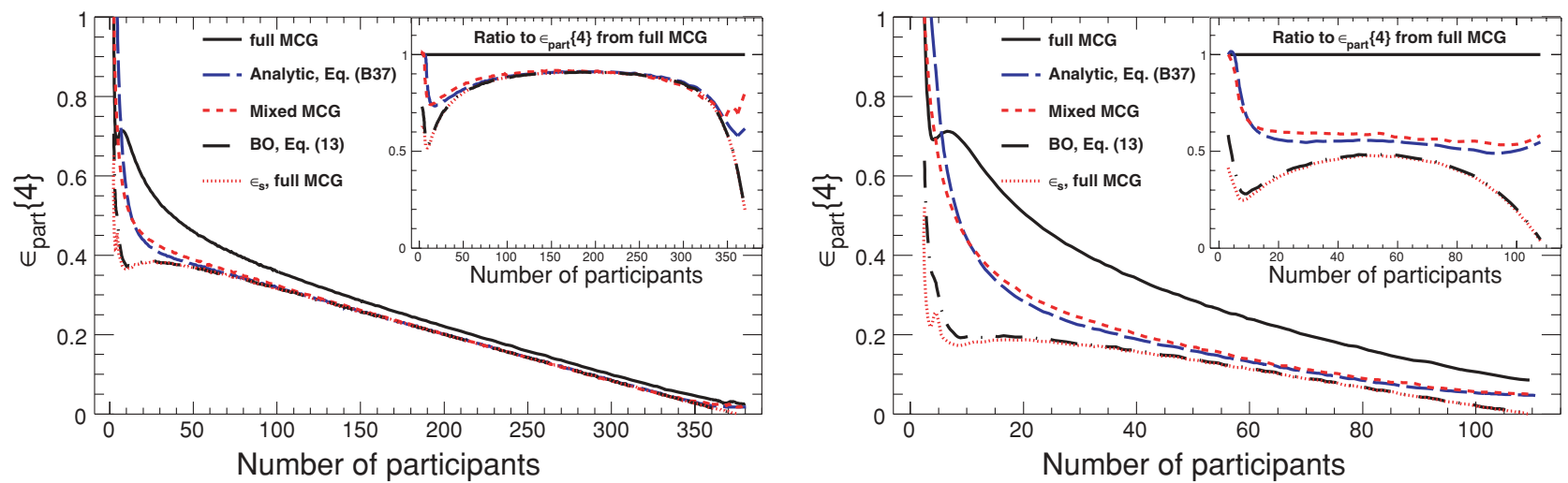

FIG. 9. (Color online) Comparison of $\epsilon_{\text {part }}\{4\}$ from the full MCG (upper set of curves), the mixed-event MCG (lower set of curves) with the semianalytical approach, Eq. (B37), and B\&O's approximation [Eq. (13) from Ref. [23]], as well as with the standard eccentricity as a function of $N_{\text {part }}$ in $\mathrm{Au}+\mathrm{Au}$ (left) and $\mathrm{Cu}+\mathrm{Cu}$ (right) collisions at $\sqrt{s_{\mathrm{NN}}}=200 \mathrm{GeV}$. The inset shows the ratio with respect to $\epsilon_{\text {part }}\{4\}$ from the full MCG calculation. 

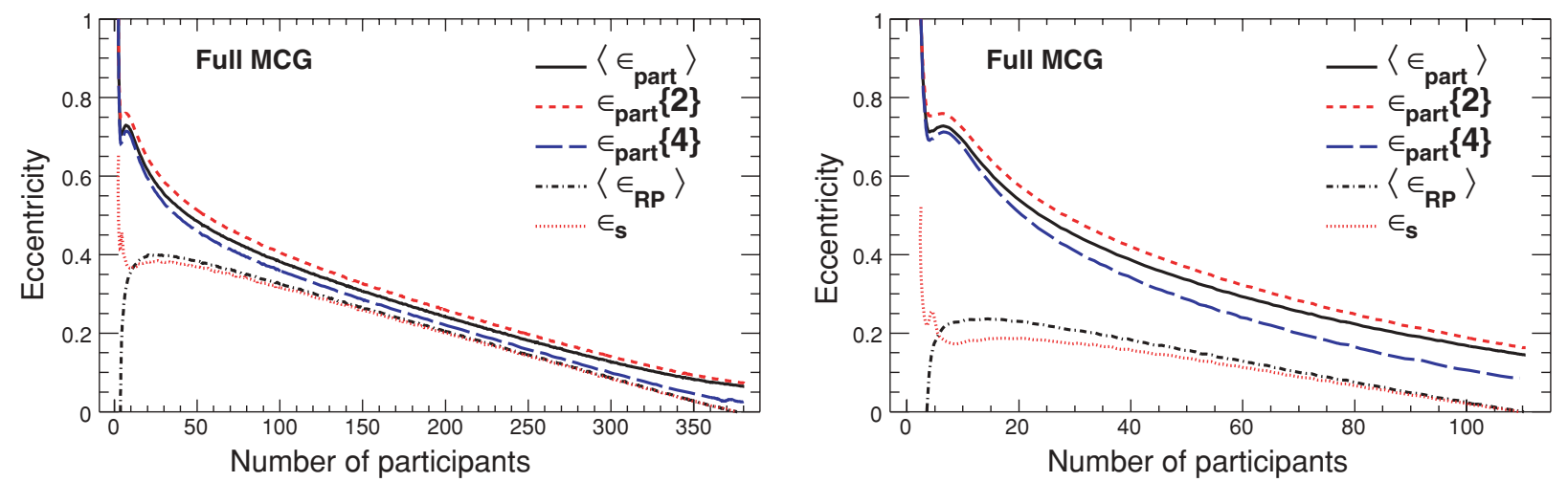

FIG. 10. (Color online) Participant eccentricity and cumulants as well as reaction-plane and standard eccentricities as functions of $N_{\text {part }}$ in $\mathrm{Au}+\mathrm{Au}$ (left) and $\mathrm{Cu}+\mathrm{Cu}$ (right) collisions at $\sqrt{s_{\mathrm{NN}}}=200 \mathrm{GeV}$ obtained from full MCG calculations.

model results are poorly described by the Bessel-Gaussian distribution given in Eq. (3) of Ref. [54] on which the equality of $\epsilon_{\text {part }}\{4\}$ and $\left\langle\epsilon_{\mathrm{RP}}\right\rangle$ is based.

\section{CONCLUSIONS}

The interpretation of the anisotropic flow data measured in nucleus-nucleus collisions at high energy requires a detailed understanding of the initial source anisotropy, which is typically quantified by the eccentricity of the shape of the nuclear overlap area. In this paper, we investigate various ways of defining this effective eccentricity using MCG calculations.

We find that variations in the Glauber parameters have only small effects on the participant eccentricity for both the $\mathrm{Au}+\mathrm{Au}$ and $\mathrm{Cu}+\mathrm{Cu}$ collision systems, while the reaction-plane eccentricity shows variations on the $10 \%$ level (Fig. 2). The generalization from participant-weighted to collision-weighted interaction point distributions leads to an increase in the obtained participant eccentricity, by a constant shift, similar to what is known for the standard eccentricity (Fig. 3). Over a realistic range of parameters, the modeling of smeared matter distributions does not lead to significantly different results for the participant eccentricity (Fig. 4). Thus, we conclude that reasonable variations in density parameters, the sources of matter, and their localization have only a small effect on the participant eccentricity. These results support our initial idea $[24,30]$ to use the participant eccentricity definition in conjunction with elliptic flow scaling. Depending on the event-plane resolution, fluctuations in the elliptic flow magnitude influence the measured "mean" $v_{2}\{\mathrm{EP}\}$, and for low resolution, they bias the measurement toward the rms of the elliptic flow distribution. For a given event-plane resolution, we find that there is a simple connection with the appropriate moment of $v_{2}$, which appears to be independent of the level of uncorrelated background (Fig. 5). For the resolutions achieved with the PHOBOS elliptic flow method [8], this gives a scaling with the rms of $v_{2}$ for all measured systems and energies. We take this into account in the eccentricity-scaled elliptic flow (Fig. 6) by presenting it as $v_{2}\{\mathrm{EP}\} / \epsilon_{\text {part }}\{2\}$ together with individual systematic errors from data and MC parameter variations. The participant-eccentricity-scaled elliptic flow shows an almost linear scaling with the particle area density as predicted in Refs. [21,22] in the low density limit.

A rigorous attempt to analytically derive nonnegligible contributions to cumulants of the participant eccentricity distribution out to $\mathcal{O}\left(1 / N_{\text {part }}^{3}\right)$ confirms the expressions found in Ref. [23], for all but $\epsilon_{\text {part }}\{4\}$, where our derivation, Eq. (B37), for the first time keeps all leading order terms in the series. The numerical evaluation of our analytical result for $\epsilon_{\text {part }}\{4\}$ agrees with $\epsilon_{\text {part }}\{4\}$ obtained with the mixed-event MCG calculation, as expected, since both ignore all correlations among the participating nucleons. In comparison, the results obtained from full PHOBOS MCG calculations, which include spatial correlations among the participants, imply that pairwise spatial correlations among the participants from the collision process itself are quite important, especially for the $\mathrm{Cu}+\mathrm{Cu}$ system. For $\mathrm{Cu}+\mathrm{Cu}$, the contribution to the participant eccentricity cumulants is about $20-45 \%$, while it is about $10 \%$ over most of the centrality range for $\mathrm{Au}+\mathrm{Au}$ (Fig. 8). Furthermore, it turns out that $\epsilon_{\text {part }}\{4\}$ for the $\mathrm{Cu}+\mathrm{Cu}$ system differs from $\epsilon_{\mathrm{s}}$ by about a factor of 2 , while the difference for $\mathrm{Au}+\mathrm{Au}$ is smaller and only about $10 \%$ for most of the centrality range (Figs. 9 and 10). Therefore, while correlations among participating nucleons may be neglected at the $10 \%$ accuracy level over a wide range of centralities in the larger $\mathrm{Au}+\mathrm{Au}$ system, they are crucially important for the smaller $\mathrm{Cu}+\mathrm{Cu}$ system and should not be neglected. These results suggest that correlations among participants that are certainly present in nature and are to a large extent implemented in full MCG calculations are sufficiently important to be taken into account in any study in which nuclear geometry is expected to play an important role.

\section{ACKNOWLEDGMENTS}

Fruitful discussions with Arthur K. Kerman, John W. Negele, and Jean-Yves Ollitrault are acknowledged. This work was partially supported by U.S. DOE Grant Nos. DE-AC02-98CH10886, DE-FG02-93ER40802, DE-FG0294ER40818, DE-FG02-94ER40865, DE-FG02-99ER41099, and DE-AC02-06CH11357; by U.S. NSF Grant Nos. 9603486 , 0072204, and 0245011; by Polish KBN Grant No. 1-P03B- 
062-27(2004-2007); by NSC of Taiwan Contract No. NSC 892112-M-008-024; and by Hungarian OTKA Grant (F 049823). The work of U.H. was supported by the U.S. DOE under Grant No. DE-FG02-01ER41190.

\section{APPENDIX A: ECCENTRICITY DEFINITIONS}

The spatial anisotropy of the interaction region in the transverse plane (in the following as throughout the paper given by the $x$ and $y$ axes) is commonly called "eccentricity" and denoted with the symbol $\epsilon$. It was introduced in Ref. [21] (called "deformation," symbol $\delta$ ) and in Ref. [14] (called "spatial asymmetry," symbol $\alpha_{x}$ ), while the basic idea of a dimensionless momentum-based anisotropy parameter (symbol $\alpha$ ) originates from Ref. [18]. In its most basic formulation, the eccentricity definition reads

$$
\epsilon=\frac{R_{y}^{2}-R_{x}^{2}}{R_{y}^{2}+R_{x}^{2}},
$$

where $R_{x}^{2}$ and $R_{y}^{2}$ characterize the size of the source in the $x$ and $y$ direction, respectively. Note that this definition allows positive and negative values, $-1 \leqslant \epsilon \leqslant 1$. It is related, but not identical, to the geometrical definition of the eccentricity of an ellipse. In this appendix, we will summarize the most important definitions of eccentricity.

\section{A. Standard eccentricity}

Prior to our work [30], it was common practice to use smooth, event-averaged initial conditions, for which the initial spatial asymmetry in the transverse plane was typically given by the "standard" eccentricity

$$
\epsilon_{\mathrm{s}}=\frac{\left\langle y^{2}\right\rangle-\left\langle x^{2}\right\rangle}{\left\langle y^{2}\right\rangle+\left\langle x^{2}\right\rangle}
$$

where $\left\langle x^{2}\right\rangle$ and $\left\langle y^{2}\right\rangle$ are the second moments of the (typically participant-weighted) ensemble-averaged nucleon distribution in the $x$ and $y$ direction, respectively. We follow the notation introduced by Bhalerao \& Ollitrault in Ref. [23], where $(. .$. denotes an average taken over many events (ensemble average) and $\{\cdots\}$ stands for an average (over participants) in a single event (sample average).

\section{B. Reaction-plane eccentricity}

The two incoming nuclei, separated by the impact parameter $b$, can be assumed to be centered at $( \pm b / 2,0)$ in the transverse plane such that for a given event, the chosen MC frame coincides with the nuclear reaction frame. The "reaction-plane" eccentricity is then obtained from

$$
\epsilon_{\mathrm{RP}}=\frac{\sigma_{y}^{2}-\sigma_{x}^{2}}{\sigma_{y}^{2}+\sigma_{x}^{2}}
$$

where $\sigma_{x}^{2}=\left\{x^{2}\right\}-\{x\}^{2}$ and $\sigma_{y}^{2}=\left\{y^{2}\right\}-\{y\}^{2}$ are the (typically participant-weighted) variances of the nucleon distribution in $x$ and $y$ direction in a given event. In contrast to $\epsilon_{\mathrm{s}}$, which is only defined for the entire ensemble of collision events, $\epsilon_{\mathrm{RP}}$ is defined for each event and has its own ensemble average $\left\langle\epsilon_{\mathrm{RP}}\right\rangle$. The reaction-plane eccentricity can be useful in comparisons with $v_{2}$ data where the reaction plane is determined by spectator neutrons in a zero-degree calorimeter [23]. Since $\left\langle\epsilon_{\mathrm{RP}}\right\rangle$ is numerically very similar to $\epsilon_{\mathrm{S}}$ (in $\mathrm{Au}+\mathrm{Au}$, the difference for $N_{\text {part }}>10$ is at most $5 \%$; however, in $\mathrm{Cu}+\mathrm{Cu}$, the difference is generally between $15 \%$ and 30\%, see also Fig. 10), it has been used in connection with MCG calculations in place of the standard eccentricity. ${ }^{4}$

\section{Participant eccentricity}

The "participant" eccentricity expresses the overlap eccentricity in the rotated (participant) frame (see Fig. 1), denoted by $x^{\prime}$ and $y^{\prime}$, which for a given event maximizes $\sigma_{y}^{\prime}$ and minimizes $\sigma_{x}^{\prime}$. In principle, the overlap zone will also be shifted with respect to the reaction-plane frame, but this shift has no impact on the eccentricity. Generally, the second moments of the position distribution in the nuclear reaction-plane (or MC) frame are described by the covariance matrix

$$
\Sigma=\left(\begin{array}{cc}
\sigma_{x}^{2} & \sigma_{x y} \\
\sigma_{x y} & \sigma_{y}^{2}
\end{array}\right),
$$

where $\sigma_{x}^{2}, \sigma_{y}^{2}$, and $\sigma_{x y}=\{x y\}-\{x\}\{y\}$ are the per-event (co-)variances of the underlying (typically participantweighted) nucleon distribution in the transverse plane, given in the original frame. The participant frame corresponds to the frame in which $\Sigma$ is diagonal. Since $\Sigma$ is a real symmetric matrix, its diagonalization can be accomplished by finding the eigenvalues $\lambda$ that satisfy $\operatorname{det}(\Sigma-\lambda I)=0$, leading to a second-order polynomial in $\lambda$ with two solutions:

$$
\lambda^{ \pm}=\frac{1}{2}\left(\sigma_{y}^{2}+\sigma_{x}^{2} \pm \sqrt{\left(\sigma_{y}^{2}-\sigma_{x}^{2}\right)^{2}+4 \sigma_{x y}^{2}}\right) .
$$

These two values of $\lambda$ correspond to $\sigma_{x}^{\prime 2}$ and $\sigma_{y}^{\prime 2}$ with the larger value $\left(\lambda^{+}\right)$corresponding to the $y^{\prime}$ and the smaller value $\left(\lambda^{-}\right)$ to the $x^{\prime}$ direction, by definition. This leads to the expression for the participant eccentricity $[24,30]$,

$$
\epsilon_{\mathrm{part}}=\frac{\sigma_{y}^{\prime 2}-\sigma_{x}^{\prime 2}}{\sigma_{y}^{\prime 2}+\sigma_{x}^{\prime 2}}=\frac{\sqrt{\left(\sigma_{y}^{2}-\sigma_{x}^{2}\right)^{2}+4 \sigma_{x y}^{2}}}{\sigma_{y}^{2}+\sigma_{x}^{2}} .
$$

Like $\epsilon_{\mathrm{RP}}$, the participant eccentricity is defined on an eventwise basis; however, in contrast to the previous definitions of the eccentricity, it is non-negative, covering the range $0 \leqslant \epsilon_{\text {part }} \leqslant 1$ by construction. The participant frame is tilted event-by-event by an angle of $\Psi_{\text {part }}$ with respect to the reaction plane, where

$$
\tan \Psi_{\text {part }}=\frac{\sigma_{x y}}{\sigma_{y}^{2}-\lambda^{-}}\left(=\frac{\sigma_{x y}}{\lambda^{+}-\sigma_{y}^{2}}\right) .
$$

It should also be noted that since the overlap ellipse is generally tilted, its area is not proportional to $\sigma_{x} \sigma_{y}$ as often assumed, but

\footnotetext{
${ }^{4}$ In Refs. [24,30] the reaction-plane eccentricity was instead named $\epsilon_{\text {std }}$ or $\epsilon_{\text {standard }}$.
} 
rather given by

$$
S=\pi \sigma_{x}^{\prime} \sigma_{y}^{\prime}=\pi \sqrt{\sigma_{x}^{2} \sigma_{y}^{2}-\sigma_{x y}^{2}} .
$$

Numerically the ratio $\frac{\sigma_{x}^{\prime} \sigma_{y}^{\prime}}{\sigma_{x} \sigma_{y}}$ is very similar for the $\mathrm{Cu}+\mathrm{Cu}$ and $\mathrm{Au}+\mathrm{Au}$ systems at the same $N_{\text {part }}$, larger than 0.75 , and increasing with increasing centrality, so for $N_{\text {part }} \geqslant 20$ it is larger than 0.9 in $200 \mathrm{GeV}$ collisions.

\section{APPENDIX B: CALCULATING CUMULANTS}

In Ref. [23], Bhalerao \& Ollitrault (B\&O) analytically derive the behavior of various cumulant moments of the participant eccentricity, making use of two questionable approximations. First, the paper contains an implicit assumption that all of the participant positions are independent samples of some underlying distribution or at least that any correlations between participants are unimportant to the eccentricity fluctuations [47]. Second, the paper uses a Taylor expansion which leads to a power series in $1 / N_{\text {part }}$ which is then truncated at $\mathcal{O}\left(1 / N_{\text {part }}\right)$ without proof that the nominally higher order terms are actually smaller.

In this appendix, we will extend the $\mathrm{B} \& \mathrm{O}$ results for the case in which any correlations between participant positions are still considered negligible, but keeping all important terms of the Taylor expansion. In particular, we will generalize Eqs. (11)-(14) of Ref. [23] and comment on Eq. (8).

\section{A. Generalizing B\&O Equation (11)}

Following B\&O, we will assume that for each event, $N \equiv N_{\text {part }}$ participants are generated independently from an arbitrary underlying two-dimensional distribution. The averaging symbol $\langle f\rangle$ denotes the average of the quantity $f$ over the underlying distribution and/or the ensemble average value taken over a large number of events. To investigate fluctuations, we must also consider eventwise averages $\{f\} \equiv \frac{1}{N} \sum_{i=1}^{N} f_{i}$. The event-by-event fluctuations are given by $\delta_{f} \equiv\{f\}-\langle f\rangle$. For convenience of calculation, let us also define $\hat{f} \equiv f-\langle f\rangle$ such that $\delta_{f}=\{\hat{f}\}$.

Obviously,

$$
\left\langle\delta_{f}\right\rangle=\langle\hat{f}\rangle=0
$$

Next, we evaluate $\left\langle\delta_{f} \delta_{g}\right\rangle$ by exhibiting the ensemble and event averages explicitly:

$$
\begin{aligned}
\left\langle\delta_{f} \delta_{g}\right\rangle & =\langle\{\hat{f}\}\{\hat{g}\}\rangle=\frac{1}{N_{e v} N^{2}} \sum_{n=1}^{N_{e v}} \sum_{i=1}^{N} \hat{f}_{i,(n)} \sum_{j=1}^{N} \hat{g}_{j,(n)} \\
& =\frac{1}{N_{e v} N^{2}}\left(\sum_{i=1}^{N} \sum_{n=1}^{N_{e v}} \hat{f}_{i,(n)} \hat{g}_{i,(n)}+\sum_{\substack{i=1 \\
j \neq i}}^{N} \sum_{n=1}^{N_{e v}} \hat{f}_{i,(n)} \hat{g}_{j,(n)}\right) \\
& =\frac{1}{N^{2}}\left(\sum_{i=1}^{N}\left\langle\hat{f}_{i} \hat{g}_{i}\right\rangle+\sum_{\substack{i=1 \\
j \neq i}}^{N}\left\langle\hat{f}_{i} \hat{g}_{j}\right\rangle\right)
\end{aligned}
$$

The sums are all finite and their order can be interchanged freely. If the participants are numbered randomly, then $\left\langle f_{i}\right\rangle=$ $\langle f\rangle$. For example, the average of the $x^{2}$ values for all "participants number 7" over all events will just be $\left\langle x^{2}\right\rangle$. Correspondingly, $\langle\hat{f}\rangle_{i}=\langle\hat{f}\rangle=0$. For $\left\langle\hat{f}_{i} \hat{g}_{i}\right\rangle$ we have

$$
\left\langle\hat{f}_{i} \hat{g}_{i}\right\rangle=\left\langle(\hat{f} \hat{g})_{i}\right\rangle=\langle\hat{f} \hat{g}\rangle .
$$

For $\left\langle\hat{f}_{i} \hat{g}_{j}\right\rangle$ with $i \neq j$ we have

$$
\left\langle\hat{f}_{i} \hat{g}_{j}\right\rangle=\left\langle\hat{f}_{i}\right\rangle\left\langle\hat{g}_{j}\right\rangle=\langle\hat{f}\rangle\langle\hat{g}\rangle=0,
$$

since, following $\mathrm{B} \& \mathrm{O}$, the positions of participants $i$ and $j(i \neq j)$ in each event are assumed to be uncorrelated. It is this last step in Eq. (B4) that fails when there are correlations between the locations of different participants. Neglecting such correlations, as in $\mathrm{B} \& \mathrm{O}$, one finally arrives at

$$
\begin{aligned}
\left\langle\delta_{f} \delta_{g}\right\rangle & =\frac{1}{N^{2}}(N\langle\hat{f} \hat{g}\rangle+N(N-1)\langle\hat{f}\rangle\langle\hat{g}\rangle) \\
& =\frac{\langle\hat{f} \hat{g}\rangle}{N}=\frac{\langle(f-\langle f\rangle)(g-\langle g\rangle)}{N} \\
& =\frac{\langle f g\rangle-\langle f\rangle\langle g\rangle}{N},
\end{aligned}
$$

in agreement with Eq. (11) from B\&O [23].

Generalizing the above derivation to higher orders, we, as well as Ollitrault [47], find that the correct generalization of Eq. (B5) for $\delta^{3}$ terms is given by

$$
\left\langle\delta_{f} \delta_{g} \delta_{h}\right\rangle=\frac{\langle\hat{f} \hat{g} \hat{h}\rangle}{N^{2}} .
$$

Compared to the $\delta^{2}$ terms, Eq. (B5), this is suppressed by a factor $1 / N$. Starting with the $\delta^{4}$ term, the expressions begin to get more complicated. In particular,

$$
\begin{aligned}
\left\langle\delta_{f} \delta_{g} \delta_{h} \delta_{u}\right\rangle= & \frac{\langle\hat{f} \hat{g} \hat{h} \hat{u}\rangle}{N^{3}}+\frac{(N-1)}{N^{3}}(\langle\hat{f} \hat{g}\rangle\langle\hat{h} \hat{u}\rangle+\langle\hat{f} \hat{h}\rangle\langle\hat{g} \hat{u}\rangle \\
& +\langle\hat{f} \hat{u}\rangle\langle\hat{g} \hat{h}\rangle),
\end{aligned}
$$

which is actually $\mathcal{O}\left(1 / N^{2}\right)$, i.e., the same order in the number of participants as the $\delta^{3}$ term.

The fifth- and sixth-order terms can be calculated similarly. Any terms involving single powers like $\langle\hat{f}\rangle$ will again vanish. The nonzero terms are

$$
\begin{aligned}
\left\langle\delta_{f} \delta_{g} \delta_{h} \delta_{u} \delta_{v}\right\rangle & \\
= & \frac{1}{N^{4}}\langle\hat{f} \hat{g} \hat{h} \hat{u} \hat{v}\rangle+\frac{N-1}{N^{4}}(\langle\hat{f} \hat{g} \hat{h}\rangle\langle\hat{u} \hat{v}\rangle+\langle\hat{f} \hat{g} \hat{u}\rangle\langle\hat{h} \hat{v}\rangle \\
& +\langle\hat{f} \hat{g} \hat{v}\rangle\langle\hat{h} \hat{u}\rangle+\langle\hat{f} \hat{h} \hat{u}\rangle\langle\hat{g} \hat{v}\rangle+\langle\hat{f} \hat{h} \hat{v}\rangle\langle\hat{g} \hat{u}\rangle+\langle\hat{f} \hat{u} \hat{v}\rangle\langle\hat{g} \hat{h}\rangle \\
& +\langle\hat{g} \hat{h} \hat{u}\rangle\langle\hat{f} \hat{v}\rangle+\langle\hat{g} \hat{h} \hat{v}\rangle\langle\hat{f} \hat{u}\rangle+\langle\hat{g} \hat{u} \hat{v}\rangle\langle\hat{f} \hat{h}\rangle+\langle\hat{h} \hat{u} \hat{v}\rangle\langle\hat{f} \hat{g}\rangle),
\end{aligned}
$$

and

$$
\begin{aligned}
\left\langle\delta_{f} \delta_{g} \delta_{h} \delta_{u} \delta_{v} \delta_{w}\right\rangle & \\
= & \frac{1}{N^{5}}[\langle\hat{f} \hat{g} \hat{h} \hat{u} \hat{v} \hat{w}\rangle+(N-1)(\langle\hat{f} \hat{g} \hat{h} \hat{u}\rangle\langle\hat{v} \hat{w}\rangle+14 \text { perms. }) \\
& +(N-1)(\langle\hat{f} \hat{g} \hat{h}\rangle\langle\hat{u} \hat{v} \hat{w}\rangle+9 \text { perms. }) \\
& +(N-1)(N-2)(\langle\hat{f} \hat{g}\rangle\langle\hat{h} \hat{u}\rangle\langle\hat{v} \hat{w}\rangle+14 \text { perms. })] .
\end{aligned}
$$


In general, the dominant terms in the $1 / N$ expansion should be those composed of products of bilinears like $\langle\hat{f} \hat{g}\rangle$ in the case of even powers of $\delta$, and those with bilinears and one trilinear in the case of odd powers of $\delta$. So, we have $\mathcal{O}\left(\delta^{2 n-1}\right)=\mathcal{O}\left(1 / N^{n}\right)$ for $n>1$ and $\mathcal{O}\left(\delta^{2 n}\right)=\mathcal{O}\left(1 / N^{n}\right)$ for $n \geqslant 1$. This means that we must consider terms up to $\mathcal{O}\left(\delta^{4}\right)$ if we want to find all $\mathcal{O}\left(1 / N^{2}\right)$ terms in the series truncated by B\&O, and terms up to $\mathcal{O}\left(\delta^{6}\right)$ in order to capture the leading behavior in the limit $\epsilon_{\mathrm{s}} \rightarrow 0$ (see below).

\section{B. B\&O Equation (8)}

The Taylor expansion which leads to Eq. (8) in $\mathrm{B} \& \mathrm{O}$ is not applicable everywhere, as it implicitly assumes that $1 /\left(N \epsilon_{\mathrm{s}}^{2}\right) \ll 1$. Since $\epsilon_{\mathrm{s}} \rightarrow 0$ for central collisions, this quantity is not guaranteed to be small, and this expansion is poorly behaved and formally divergent for central collisions. Fortunately, Eq. (8) of B\&O is not actually needed to derive and generalize their Eqs. (12)-(14).

\section{B\&O Equation (12): Calculating $\epsilon_{\text {part }}\{2\}^{2}$}

To calculate $\epsilon_{\text {part }}\{2\}^{2}=\left\langle\epsilon_{\text {part }}^{2}\right\rangle$, we must first express $\epsilon_{\text {part }}^{2}$ in terms of the $\delta$ 's. We start with the definition, Eq. (A6):

$$
\epsilon_{\text {part }}^{2}=\frac{\left(\sigma_{y}^{2}-\sigma_{x}^{2}\right)^{2}+4 \sigma_{x y}^{2}}{\left(\sigma_{y}^{2}+\sigma_{x}^{2}\right)^{2}} .
$$

Following B\&O, we have

$$
\begin{aligned}
\sigma_{x}^{2} & =\left\{x^{2}\right\}-\{x\}^{2}=\left\langle x^{2}\right\rangle+\delta_{x^{2}}-\delta_{x}^{2}, \\
\sigma_{y}^{2} & =\left\{y^{2}\right\}-\{y\}^{2}=\left\langle y^{2}\right\rangle+\delta_{y^{2}}-\delta_{y}^{2}, \\
\sigma_{x y} & =\{x y\}-\{x\}\{y\}=\delta_{x y}-\delta_{x} \delta_{y},
\end{aligned}
$$

using $\langle x\rangle=\langle y\rangle=\langle x y\rangle=\left\langle x y^{n}\right\rangle=\left\langle x^{n} y\right\rangle=0$. This leads to the exact result

$$
\begin{aligned}
\epsilon_{\text {part }}^{2}= & {\left[\epsilon_{\mathrm{s}}^{2}+\frac{2 \epsilon_{\mathrm{s}} \delta_{y^{2}-x^{2}}}{\left\langle r^{2}\right\rangle}+\frac{\delta_{y^{2}-x^{2}}^{2}}{\left\langle r^{2}\right\rangle^{2}}+\frac{4 \delta_{x y}^{2}}{\left\langle r^{2}\right\rangle^{2}}-\frac{2 \epsilon_{\mathrm{s}}\left(\delta_{y}^{2}-\delta_{x}^{2}\right)}{\left\langle r^{2}\right\rangle}\right.} \\
& -\frac{2 \delta_{y^{2}-x^{2}}\left(\delta_{y}^{2}-\delta_{x}^{2}\right)}{\left\langle r^{2}\right\rangle^{2}}-\frac{8 \delta_{x y} \delta_{x} \delta_{y}}{\left\langle r^{2}\right\rangle^{2}}+\frac{\left(\delta_{y}^{2}-\delta_{x}^{2}\right)^{2}}{\left\langle r^{2}\right\rangle^{2}} \\
& \left.+\frac{4 \delta_{x}^{2} \delta_{y}^{2}}{\left\langle r^{2}\right\rangle^{2}}\right]\left[1+\frac{\delta_{r^{2}}}{\left\langle r^{2}\right\rangle}-\frac{\left(\delta_{y}^{2}+\delta_{x}^{2}\right)}{\left\langle r^{2}\right\rangle}\right]^{-2},
\end{aligned}
$$

where $\left\langle r^{2}\right\rangle=\left\langle x^{2}\right\rangle+\left\langle y^{2}\right\rangle$. The second and third terms in the denominator are genuinely $\ll 1$, so it can be safely Taylor expanded. The resulting polynomial series is well behaved. The leading terms are $\epsilon_{\mathrm{s}}^{2}$ and $\mathcal{O}\left(\epsilon_{\mathrm{s}}^{0} / N\right)$. All terms of $\mathcal{O}\left(\delta^{3}\right)$ and higher are $\mathcal{O}\left(\epsilon_{\mathrm{s}}^{0} / N^{2}\right)$ and can be dropped, since they are at least a full power of $1 / N$ down without any compensating $1 / \epsilon_{\mathrm{s}}$ factors. So, we obtain

$$
\begin{aligned}
\epsilon_{\mathrm{part}}^{2}= & \epsilon_{\mathrm{s}}^{2}+\frac{2 \epsilon_{\mathrm{s}} \delta_{y^{2}-x^{2}}}{\left\langle r^{2}\right\rangle}-\frac{2 \epsilon_{\mathrm{s}}^{2} \delta_{r^{2}}}{\left\langle r^{2}\right\rangle}+\frac{\delta_{y^{2}-x^{2}}^{2}}{\left\langle r^{2}\right\rangle^{2}}+\frac{4 \delta_{x y}^{2}}{\left\langle r^{2}\right\rangle^{2}} \\
& -\frac{2 \epsilon_{\mathrm{s}}\left(\delta_{y}^{2}-\delta_{x}^{2}\right)}{\left\langle r^{2}\right\rangle}-\frac{4 \epsilon_{\mathrm{s}} \delta_{r^{2}} \delta_{y^{2}-x^{2}}}{\left\langle r^{2}\right\rangle^{2}}+\frac{2 \epsilon_{\mathrm{s}}^{2}\left(\delta_{y}^{2}+\delta_{x}^{2}\right)}{\left\langle r^{2}\right\rangle}
\end{aligned}
$$

$$
\begin{aligned}
& +\frac{3 \epsilon_{\mathrm{s}}^{2} \delta_{r^{2}}^{2}}{\left\langle r^{2}\right\rangle^{2}}+\mathcal{O}\left(\epsilon_{\mathrm{s}}^{0} \delta^{3}\right)+\mathcal{O}\left(\epsilon_{\mathrm{s}}^{0} \delta^{4}\right) \\
& +\mathcal{O}\left(\epsilon_{\mathrm{s}}^{0} \delta^{5}\right)+\mathcal{O}\left(\epsilon_{\mathrm{s}}^{0} \delta^{6}\right)+\cdots .
\end{aligned}
$$

This leads to the same result as B\&O Eq. (12), except that we have also shown that all further terms are subdominant:

$$
\begin{aligned}
\left\langle\epsilon_{\mathrm{part}}^{2}\right\rangle= & \epsilon_{\mathrm{s}}^{2}+\frac{1}{N\left\langle r^{2}\right\rangle^{2}}\left[\left(1+3 \epsilon_{\mathrm{s}}^{2}\right)\left\langle r^{4}\right\rangle+4 \epsilon_{\mathrm{s}}\left\langle r^{4} \cos 2 \phi\right\rangle\right] \\
& +\mathcal{O}\left(\frac{\epsilon_{\mathrm{s}}^{0}}{N^{2}}\right)+\cdots .
\end{aligned}
$$

Similarly, Eq. (14) in B\&O is well behaved and correctly contains all of the leading terms. As noted above, this is different for the expansion of Eq. (8) in $\mathrm{B} \& \mathrm{O}$, which does not converge in the limit $\epsilon_{\mathrm{s}} \rightarrow 0$.

\section{B\&O Equation (13): Calculating $\epsilon_{\text {part }}\{4\}^{4}$}

We know from $\mathrm{B} \& \mathrm{O}$ that the $\mathcal{O}\left(\epsilon_{\mathrm{s}}^{2} / N\right)$ terms cancel, leaving the $\epsilon_{\mathrm{s}}^{4}$ term as apparently dominant. However, to confirm this, we need to check that all of the nominally higher order terms are actually small.

To organize the calculation, let us write the expansion of Eq. (B14) as

$$
\epsilon_{\mathrm{part}}^{2}=\epsilon_{\mathrm{s}}^{2}+\mathcal{A}+B+C+D+\cdots,
$$

where $\mathcal{A}$ contains all terms of $\mathcal{O}(\delta), \mathcal{B}$ of $\mathcal{O}\left(\delta^{2}\right)$, and so on. Furthermore, let us define $\mathcal{B}_{0}=\lim _{\epsilon_{\mathrm{s}} \rightarrow 0} \mathcal{B}$ and $\mathcal{C}_{0}=$ $\lim _{\epsilon_{\mathrm{s}} \rightarrow 0} \mathcal{C}$, etc., so that

$$
\lim _{\epsilon_{\mathrm{s}} \rightarrow 0} \epsilon_{\mathrm{part}}^{2}=\mathcal{B}_{0}+\mathcal{C}_{0}+\mathcal{D}_{0} .
$$

Explicitly, we will need the following equations:

$$
\begin{aligned}
\mathcal{A} \equiv & \frac{2 \epsilon_{\mathrm{s}} \delta_{y^{2}-x^{2}}}{\left\langle r^{2}\right\rangle}-\frac{2 \epsilon_{\mathrm{s}}^{2} \delta_{r^{2}}}{\left\langle r^{2}\right\rangle}, \\
\mathcal{B} \equiv & \frac{\delta_{y^{2}-x^{2}}^{2}+4 \delta_{x y}^{2}}{\left\langle r^{2}\right\rangle^{2}}-\frac{2 \epsilon_{\mathrm{s}}\left(\delta_{y}^{2}-\delta_{x}^{2}\right)}{\left\langle r^{2}\right\rangle}-\frac{4 \epsilon_{\mathrm{s}} \delta_{r^{2}} \delta_{y^{2}-x^{2}}}{\left\langle r^{2}\right\rangle^{2}} \\
& +\frac{2 \epsilon_{\mathrm{s}}^{2}\left(\delta_{y}^{2}+\delta_{x}^{2}\right)}{\left\langle r^{2}\right\rangle}+\frac{3 \epsilon_{\mathrm{s}}^{2} \delta_{r^{2}}^{2}}{\left\langle r^{2}\right\rangle^{2}}, \\
\mathcal{C} \equiv & -\frac{2 \delta_{y^{2}-x^{2}}\left(\delta_{y}^{2}-\delta_{x}^{2}\right)}{\left\langle r^{2}\right\rangle^{2}}-\frac{8 \delta_{x y} \delta_{x} \delta_{y}}{\left\langle r^{2}\right\rangle^{2}}-\frac{2 \delta_{y^{2}-x^{2}}^{2} \delta_{r^{2}}}{\left\langle r^{2}\right\rangle^{3}} \\
& -\frac{8 \delta_{x y}^{2} \delta_{r^{2}}}{\left\langle r^{2}\right\rangle^{3}}+\frac{4 \epsilon_{\mathrm{s}} \delta_{r^{2}}\left(\delta_{y}^{2}-\delta_{x}^{2}\right)}{\left\langle r^{2}\right\rangle^{2}}+\frac{4 \epsilon_{\mathrm{s}} \delta_{y^{2}-x^{2}}\left(\delta_{y}^{2}+\delta_{x}^{2}\right)}{\left\langle r^{2}\right\rangle^{2}} \\
& +\frac{6 \epsilon_{\mathrm{s}} \delta_{r^{2}}^{2} \delta_{y^{2}-x^{2}}}{\left\langle r^{2}\right\rangle^{3}}-\frac{6 \epsilon_{\mathrm{s}}^{2} \delta_{r^{2}}\left(\delta_{y}^{2}+\delta_{x}^{2}\right)}{\left\langle r^{2}\right\rangle^{2}}-\frac{4 \epsilon_{\mathrm{s}}^{2} \delta_{r^{2}}^{3}}{\left\langle r^{2}\right\rangle^{3}}, \quad(\mathrm{~B} 21) \\
\mathcal{B}_{0} \equiv & \frac{\delta_{y^{2}-x^{2}}^{2}+4 \delta_{x y}^{2}}{\left\langle r^{2}\right\rangle^{2}}, \quad(\mathrm{~B} 22) \\
\mathcal{C}_{0} \equiv & -\frac{2 \delta_{y^{2}-x^{2}}\left(\delta_{y}^{2}-\delta_{x}^{2}\right)}{\left\langle r^{2}\right\rangle^{2}}-\frac{8 \delta_{x y} \delta_{x} \delta_{y}}{\left\langle r^{2}\right\rangle^{2}}-\frac{2\left(\delta_{y^{2}-x^{2}}^{2}+4 \delta_{x y}^{2}\right) \delta_{r^{2}}}{\left\langle r^{2}\right\rangle^{3}},
\end{aligned}
$$




$$
\begin{aligned}
\mathcal{D}_{0} \equiv & \frac{\left(\delta_{y}^{2}+\delta_{x}^{2}\right)^{2}}{\left\langle r^{2}\right\rangle^{2}}+\frac{4 \delta_{r^{2}} \delta_{y^{2}-x^{2}}\left(\delta_{y}^{2}-\delta_{x}^{2}\right)}{\left\langle r^{2}\right\rangle^{3}}+\frac{16 \delta_{x y} \delta_{x} \delta_{y} \delta_{r^{2}}}{\left\langle r^{2}\right\rangle^{3}} \\
& +\frac{2\left(\delta_{y^{2}-x^{2}}^{2}+4 \delta_{x y}^{2}\right)\left(\delta_{y}^{2}+\delta_{x}^{2}\right)}{\left\langle r^{2}\right\rangle^{3}}+\frac{3\left(\delta_{y^{2}-x^{2}}^{2}+4 \delta_{x y}^{2}\right) \delta_{r^{2}}^{2}}{\left\langle r^{2}\right\rangle^{4}}
\end{aligned}
$$

We can now calculate $\epsilon_{\text {part }}\{4\}^{4}$ as

$$
\begin{aligned}
\epsilon_{\mathrm{part}}\{4\}^{4} \equiv & 2\left\langle\epsilon_{\mathrm{part}}^{2}\right\rangle^{2}-\left\langle\epsilon_{\mathrm{part}}^{4}\right\rangle \\
= & \epsilon_{\mathrm{s}}^{4}-\langle\mathcal{A}\rangle^{2}+2 \epsilon_{\mathrm{s}}^{2}\langle\mathcal{B}\rangle+2 \epsilon_{\mathrm{s}}^{2}\langle\mathcal{C}\rangle_{0}-2\langle\mathcal{A B}\rangle \\
& +2 \epsilon_{\mathrm{s}}^{2}\langle\mathcal{D}\rangle_{0}-2\langle\mathcal{A C}\rangle+2\langle\mathcal{B}\rangle^{2}-\langle\mathcal{B}\rangle^{2} \\
& +4\langle\mathcal{B}\rangle_{0}\langle\mathcal{C}\rangle_{0}-2\left\langle\mathcal{B}_{0} \mathcal{C}_{0}\right\rangle+4\langle\mathcal{B}\rangle_{0}\langle\mathcal{D}\rangle_{0} \\
& -2\left\langle\mathcal{B}_{0} \mathcal{D}_{0}\right\rangle-\langle\mathcal{C}\rangle_{0}^{2}
\end{aligned}
$$

where we have kept all terms up to $\mathcal{O}(1 / N)$ and the leading terms in $\epsilon_{\mathrm{s}}$ at $\mathcal{O}\left(1 / N^{2}\right)$ and $\mathcal{O}\left(1 / N^{3}\right)$. We evaluate the expressions in Eq. (B25) piece by piece, dropping any terms that would contribute to $\epsilon_{\text {part }}\{4\}^{4}$ at $\mathcal{O}\left(\epsilon_{\mathrm{s}}^{4} / N^{2}\right)$ or $\mathcal{O}\left(\epsilon_{\mathrm{s}}^{n} / N^{3}\right)$ for each $n>0$. Note that $\left\langle r^{m} \cos 2 n \phi\right\rangle=\mathcal{O}\left(\epsilon_{\mathrm{s}}^{n}\right)$. This leads to the following expressions:

$$
\begin{aligned}
\langle\mathcal{B}\rangle= & \frac{1}{N\left\langle r^{2}\right\rangle^{2}}\left[\left(1+3 \epsilon_{\mathrm{s}}^{2}\right)\left\langle r^{4}\right\rangle+4 \epsilon_{\mathrm{s}}\left\langle r^{4} \cos 2 \phi\right\rangle\right], \\
\langle\mathcal{B}\rangle_{0}= & \frac{\left\langle r^{4}\right\rangle}{N\left\langle r^{2}\right\rangle^{2}}, \\
\langle\mathcal{A}\rangle^{2}= & \frac{1}{N\left\langle r^{2}\right\rangle^{2}}\left[\left(2 \epsilon_{\mathrm{s}}^{2}+4 \epsilon_{\mathrm{s}}^{4}\right)\left\langle r^{4}\right\rangle+8 \epsilon_{\mathrm{s}}^{3}\left\langle r^{4} \cos 2 \phi\right\rangle\right. \\
& \left.+2 \epsilon_{\mathrm{s}}^{2}\left\langle r^{4} \cos 4 \phi\right\rangle\right], \\
\langle\mathcal{C}\rangle_{0}= & \frac{1}{N^{2}}\left[-\frac{2\left\langle r^{6}\right\rangle}{\left\langle r^{2}\right\rangle^{3}}\right], \\
\langle\mathcal{A B}\rangle= & \frac{1}{N^{2}}\left[-6 \epsilon_{\mathrm{s}}^{2} \frac{\left\langle r^{6}\right\rangle}{\left\langle r^{2}\right\rangle^{3}}-2 \epsilon_{\mathrm{s}} \frac{\left\langle r^{6} \cos 2 \phi\right\rangle}{\left\langle r^{2}\right\rangle^{3}}\right], \\
\langle\mathcal{D}\rangle_{0}= & \frac{1}{N^{2}}\left[2-\frac{\left\langle r^{4}\right\rangle}{\left\langle r^{2}\right\rangle^{2}}+\frac{3\left\langle r^{4}\right\rangle^{2}}{\left\langle r^{2}\right\rangle^{4}}\right], \\
\langle\mathcal{A C}\rangle= & \frac{1}{N^{2}\left\langle r^{2}\right\rangle^{4}}\left[10 \epsilon_{\mathrm{s}}^{2}\left\langle r^{4}\right\rangle^{2}+8 \epsilon_{\mathrm{s}}\left\langle r^{4}\right\rangle\left\langle r^{4} \cos 2 \phi\right\rangle\right]
\end{aligned}
$$

$$
\begin{aligned}
\langle\mathcal{B}\rangle^{2}= & \frac{1}{N^{2}\left\langle r^{2}\right\rangle^{4}}\left[\left(1+6 \epsilon_{\mathrm{s}}^{2}\right)\left\langle r^{4}\right\rangle^{2}+8 \epsilon_{\mathrm{s}}\left\langle r^{4}\right\rangle\left\langle r^{4} \cos 2 \phi\right\rangle\right], \\
\left\langle\mathcal{B}^{2}\right\rangle= & \frac{1}{N^{2}}\left[4 \epsilon_{\mathrm{s}}^{2}-2 \epsilon_{\mathrm{s}}^{2} \frac{\left\langle r^{4}\right\rangle}{\left\langle r^{2}\right\rangle^{2}}+\left(2+14 \epsilon_{\mathrm{s}}^{2}\right) \frac{\left\langle r^{4}\right\rangle^{2}}{\left\langle r^{2}\right\rangle^{4}}\right. \\
& \left.+16 \epsilon_{\mathrm{s}} \frac{\left\langle r^{4}\right\rangle\left\langle r^{4} \cos 2 \phi\right\rangle}{\left\langle r^{2}\right\rangle^{4}}\right]+\frac{\left\langle r^{8}\right\rangle-2\left\langle r^{4}\right\rangle^{2}}{N^{3}\left\langle r^{2}\right\rangle^{4}} \\
\left\langle\mathcal{B}_{0} \mathcal{C}_{0}\right\rangle= & \frac{1}{N^{3}}\left[\frac{4\left\langle r^{4}\right\rangle^{2}}{\left\langle r^{2}\right\rangle^{4}}-\frac{8\left\langle r^{4}\right\rangle\left\langle r^{6}\right\rangle}{\left\langle r^{2}\right\rangle^{5}}\right], \\
\left\langle\mathcal{B}_{0} \mathcal{D}_{0}\right\rangle= & \frac{1}{N^{3}}\left[\frac{2\left\langle r^{4}\right\rangle}{\left\langle r^{2}\right\rangle^{2}}-\frac{2\left\langle r^{4}\right\rangle^{2}}{\left\langle r^{2}\right\rangle^{4}}+\frac{6\left\langle r^{4}\right\rangle^{3}}{\left\langle r^{2}\right\rangle^{6}}\right] \\
\left\langle\mathcal{C}_{0}^{2}\right\rangle= & \frac{1}{N^{3}}\left[\frac{4\left\langle r^{4}\right\rangle}{\left\langle r^{2}\right\rangle^{2}}-\frac{8\left\langle r^{4}\right\rangle^{2}}{\left\langle r^{2}\right\rangle^{4}}+\frac{8\left\langle r^{4}\right\rangle^{3}}{\left\langle r^{2}\right\rangle^{6}}\right]
\end{aligned}
$$

Assembling, this leads to the final result

$$
\begin{aligned}
\epsilon_{\mathrm{part}}\{4\}^{4}= & \epsilon_{\mathrm{s}}^{4}+\frac{1}{N\left\langle r^{2}\right\rangle^{2}}\left[2 \epsilon_{\mathrm{s}}^{4}\left\langle r^{4}\right\rangle-2 \epsilon_{\mathrm{s}}^{2}\left\langle r^{4} \cos 4 \phi\right\rangle\right] \\
& +\frac{1}{N^{2}}\left[8 \epsilon_{\mathrm{s}}^{2} \frac{\left\langle r^{6}\right\rangle}{\left\langle r^{2}\right\rangle^{3}}+4 \epsilon_{\mathrm{s}} \frac{\left\langle r^{6} \cos 2 \phi\right\rangle}{\left\langle r^{2}\right\rangle^{3}}\right. \\
& \left.-16 \epsilon_{\mathrm{s}}^{2} \frac{\left\langle r^{4}\right\rangle^{2}}{\left\langle r^{2}\right\rangle^{4}}-16 \epsilon_{\mathrm{s}} \frac{\left\langle r^{4}\right\rangle\left\langle r^{4} \cos 2 \phi\right\rangle}{\left\langle r^{2}\right\rangle^{4}}\right] \\
& +\frac{1}{N^{3}}\left[\frac{2\left\langle r^{4}\right\rangle^{2}}{\left\langle r^{2}\right\rangle^{4}}-\frac{\left\langle r^{8}\right\rangle}{\left\langle r^{2}\right\rangle^{4}}+\frac{8\left\langle r^{4}\right\rangle\left\langle r^{6}\right\rangle}{\left\langle r^{2}\right\rangle^{5}}-\frac{8\left\langle r^{4}\right\rangle^{3}}{\left\langle r^{2}\right\rangle^{6}}\right] \\
& +\mathcal{O}\left(\frac{\epsilon_{\mathrm{s}}^{4}}{N^{2}}\right)+\mathcal{O}\left(\frac{\epsilon_{\mathrm{s}}^{2}}{N^{3}}\right) \\
& +\mathcal{O}\left(\frac{\epsilon_{\mathrm{s}}^{0}}{N^{4}}\right)+\mathcal{O}\left(\frac{\epsilon_{\mathrm{s}}^{0}}{N^{5}}\right)+\cdots,
\end{aligned}
$$

where we now have all of the leading terms. Terms which have been dropped are down from the leading terms by at least a full factor of $1 / N$ without any compensating $1 / \epsilon_{\mathrm{s}}$ factor. $\mathrm{B} \& \mathrm{O}$ [23] left out the $\mathcal{O}\left(1 / N^{2}\right)$ term and most importantly the $\mathcal{O}\left(1 / N^{3}\right)$ term. For $\mathrm{Cu}+\mathrm{Cu}$, the $\mathcal{O}\left(1 / N^{3}\right)$ term tends to be comparable to the "leading" $\epsilon_{\mathrm{s}}^{4}$ term. For central collisions, as $\epsilon_{\mathrm{s}}$ vanishes, the $\mathcal{O}\left(1 / N^{3}\right)$ term becomes dominant and certainly cannot be neglected.
[1] I. Arsene et al. (BRAHMS Collaboration), Nucl. Phys. A757, 1 (2005).

[2] K. Adcox et al. (PHENIX Collaboration), Nucl. Phys. A757, 184 (2005).

[3] B. B. Back et al. (PHOBOS Collaboration), Nucl. Phys. A757, 28 (2005).

[4] J. Adams et al. (STAR Collaboration), Nucl. Phys. A757, 102 (2005).

[5] K. Adcox et al. (PHENIX Collaboration), Phys. Rev. Lett. 89, 212301 (2002).

[6] S. S. Adler et al. (PHENIX Collaboration), Phys. Rev. Lett. 91, 182301 (2003).

[7] S. S. Adler et al. (PHENIX Collaboration), Phys. Rev. Lett. 94, 232302 (2005).
[8] B. B. Back et al. (PHOBOS Collaboration), Phys. Rev. Lett. 89, 222301 (2002).

[9] B. B. Back et al. (PHOBOS Collaboration), Phys. Rev. C 72, 051901 (2005).

[10] B. B. Back et al. (PHOBOS Collaboration), Phys. Rev. Lett. 94, 122303 (2005).

[11] K. H. Ackermann et al. (STAR Collaboration), Phys. Rev. Lett. 86, 402 (2001).

[12] J. Adams et al. (STAR Collaboration), Phys. Rev. C 72, 014904 (2005).

[13] H. Sorge, Phys. Rev. Lett. 78, 2309 (1997).

[14] H. Sorge, Phys. Rev. Lett. 82, 2048 (1999).

[15] P. F. Kolb, J. Sollfrank, and U. Heinz, Phys. Rev. C 62, 054909 (2000). 
[16] U. Heinz and P. F. Kolb, Nucl. Phys. A702, 269 (2002).

[17] A. M. Poskanzer and S. A. Voloshin, Phys. Rev. C 58, 1671 (1998).

[18] J.-Y. Ollitrault, Phys. Rev. D 46, 229 (1992).

[19] P. F. Kolb, P. Huovinen, U. Heinz, and H. Heiselberg, Phys. Lett. B500, 232 (2001).

[20] R. S. Bhalerao, J.-P. Blaizot, N. Borghini, and J.-Y. Ollitrault, Phys. Lett. B627, 49 (2005).

[21] H. Heiselberg and A.-M. Levy, Phys. Rev. C 59, 2716 (1999).

[22] S. A. Voloshin and A. M. Poskanzer, Phys. Lett. B474, 27 (2000).

[23] R. S. Bhalerao and J.-Y. Ollitrault, Phys. Lett. B641, 260 (2006).

[24] B. Alver et al. (PHOBOS Collaboration), Phys. Rev. Lett. 98, 242302 (2007).

[25] A. Adare et al. (PHENIX Collaboration), Phys. Rev. Lett. 98, 162301 (2007).

[26] T. Hirano, M. Isse, Y. Nara, A. Ohnishi, and K. Yoshino, Phys. Rev. C 72, 041901 (2005).

[27] B. Alver et al. (PHOBOS Collaboration), Phys. Rev. Lett. 96, 212301 (2006).

[28] G. Roland et al. (PHOBOS Collaboration), Nucl. Phys. A774, 113 (2006).

[29] M. Miller and R. Snellings, arXiv:nucl-ex/0312008.

[30] S. Manly et al. (PHOBOS Collaboration), Nucl. Phys. A774, 523 (2006).

[31] B. Alver et al. (PHOBOS Collaboration), J. Phys. G 34, S887 (2007).

[32] B. Alver et al. (PHOBOS Collaboration), arXiv:nuclex/0702036.

[33] P. Sorensen (STAR Collaboration), J. Phys. G 34, S897 (2007).

[34] M. L. Miller, K. Reygers, S. J. Sanders, and P. Steinberg, Ann. Rev. Nucl. Part. Sci. 57, 205 (2007).
[35] A. Bialas, M. Bleszynski, and W. Czyz, Nucl. Phys. B111, 461 (1976).

[36] A. Bialas, M. Bleszynski, and W. Czyz, Acta Phys. Pol. B 8, 389 (1977).

[37] T. W. Ludlam, A. Pfoh, and A. Shor (1986), Brookhaven National Laboratory Report, BNL-37196, Feb. 1986 (unpublished).

[38] A. Shor and R. S. Longacre, Phys. Lett. B218, 100 (1989).

[39] W. M. Yao et al. (Particle Data Group), J. Phys. G 33, 1 (2006).

[40] C. W. De Jager, H. De Vries, and C. De Vries, At. Data Nucl. Data Tables 36, 495 (1987).

[41] X.-N. Wang and M. Gyulassy, Phys. Rev. D 44, 3501 (1991).

[42] B. B. Back et al. (PHOBOS Collaboration), Phys. Rev. C 70, 021902 (2004).

[43] D. Kharzeev and M. Nardi, Phys. Lett. B507, 121 (2001).

[44] P. F. Kolb, U. W. Heinz, P. Huovinen, K. J. Eskola, and K. Tuominen, Nucl. Phys. A696, 197 (2001).

[45] W. Broniowski, P. Bozek, and M. Rybczynski, Phys. Rev. C 76, 054905 (2007).

[46] N. Borghini, P. M. Dinh, and J.-Y. Ollitrault, Phys. Rev. C 64, 054901 (2001).

[47] J.-Y. Ollitrault (private communication).

[48] C. Adler et al. (STAR Collaboration), Phys. Rev. C 66, 034904 (2002).

[49] B. B. Back et al. (PHOBOS Collaboration), Phys. Rev. C 65, 061901 (2002).

[50] B. B. Back et al. (PHOBOS Collaboration), Phys. Rev. C 74, 021901 (2006).

[51] B. Alver et al. (PHOBOS Collaboration), arXiv:0709.4008 [nucl-ex].

[52] W. Czyz and L. C. Maximon, Ann. Phys. (NY) 52, 59 (1969).

[53] A. Białas and W. Czyz (private communication).

[54] S. A. Voloshin, A. M. Poskanzer, A. Tang, and G. Wang, Phys. Lett. B659, 537 (2008). 